\title{
Altered hippocampal kynurenine pathway metabolism contribute to hyperexcitability in mesial temporal lobe epilepsy-hippocampal sclerosis
}

\author{
Soumil Dey ${ }^{1}$, Aparna Banerjee Dixit ${ }^{2}$, Manjari Tripathi ${ }^{1}$, Ramesh Doddamani ${ }^{1}$, Meher \\ Sharma $^{1}$, Sanjeev Lalwani ${ }^{1}$, Sarat Chandra ${ }^{1}$, and Jyotirmoy Banerjee ${ }^{1}$ \\ ${ }^{1}$ All India Institute of Medical Sciences \\ ${ }^{2}$ University of Delhi
}

September 28, 2020

\begin{abstract}
Background and Purpose Glutamate receptor mediated enhanced excitatory neurotransmission is typically associated with mesial temporal lobe epilepsy with hippocampal sclerosis (MTLE-HS). Kynurenic acid (KYNA) and quinolinic acid (QUIN) are two important tryptophan-kynurenine pathway (KP) metabolites that modulate glutamate receptor activity. This study was designed to test the hypothesis that alteration in metabolism of KP metabolites in the hippocampus of patients with MTLEHS contributes to abnormal glutamatergic transmission. Experimental Approach TKP metabolites level were determined using HPLC and LC-MS/MS in the hippocampal samples of patients with MTLE-HS compared to autopsy and non-seizure control samples. mRNA and protein expression of TKP enzymes were determined by qPCR and western blot. Spontaneous glutamatergic activities were recorded from pyramidal neurons in presence of kynurenine (KYN) and KYNA using whole cell patch clamp. Key Results We observed significantly reduced KYNA and elevated QUIN levels in the hippocampal samples, while KYN level remains unaltered. Spontaneous glutamatergic activity in the hippocampal samples was higher compared to that in non-seizure controls. Treatment with kynurenine inhibited the glutamatergic activity in non-seizure control samples but not in case of the hippocampal samples. However, exogenously applied KYNA inhibited glutamatergic activity in both non-seizure control and hippocampal samples. We also observed reduced levels of enzyme kynurenine aminotransferase II and its co-factor pyridoxal phosphate in the hippocampal samples. Conclusion Our findings indicate that altered metabolism of TKP metabolites in hippocampus could contribute to hyperglutamatergic tone in patients with MTLE-HS.
\end{abstract}

Title: Altered hippocampal kynurenine pathway metabolism contribute to hyperexcitability in mesial temporal lobe epilepsy-hippocampal sclerosis

Running title: Altered kynurenine pathway metabolism in hippocampal sclerosis

Authors: Soumil Dey ${ }^{1}$, Aparna Banerjee Dixit ${ }^{2}$, Manjari Tripathi ${ }^{3}$, Ramesh S. Doddamani ${ }^{1}$, Meher C. Sharma $^{4}$, Sanjeev Lalwani ${ }^{5}$, P. Sarat Chandra ${ }^{1^{*}}$, Jyotirmoy Banerjee $6^{6^{*}}$

1 Department of Neurosurgery, All India Institute of Medical Sciences, New Delhi, India

2 Dr. B. R. Ambedkar Centre for Biomedical Research, University of Delhi, New Delhi, India

3 Department of Neurology, All India Institute of Medical Sciences, New Delhi, India

4 Department of Pathology, All India Institute of Medical Sciences, New Delhi, India

5 Department of Forensic Medicine and Toxicology, All India Institute of Medical Sciences, New Delhi, India 6 Department of Biophysics, All India Institute of Medical Sciences, New Delhi, India 
*Corresponding author

Jyotirmoy Banerjee

Department of Biophysics

All India Institute of Medical Sciences (AIIMS), New Delhi, India-110029

Telephone: +91-11-26549321, Email: jyotirmoybanerjee1@gmail.com

P Sarat Chandra

Department of Neurosurgery

All India Institute of Medical Sciences (AIIMS), New Delhi, India. 110029.

Telephone: +91-11-26546477, E-mail: saratpchandra3@gmail.com

\section{Author contribution:}

$\mathrm{SD}, \mathrm{ABD}, \mathrm{MT}, \mathrm{PSC}$ and JB designed the research, performed experiments, analysed data and wrote the manuscript. MT recruited MTLE-HS patients for surgery, RD and PSC performed surgery on patients and provided brain specimens. MCS performed neuropathological examination. SL provided autopsy control tissues. All authors read and approved the final manuscript.

\section{Word count}

Introduction: 540

Methods: 2377

Results: 1934

Discussion and conclusion: 1390

\section{Data Availability Statement}

The data that support the findings of this study are available from the corresponding author upon reasonable request. Some data may not be made available because of privacy or ethical restrictions.

\section{Acknowledgment of funding}

This work was financially supported by Grant No: BT/MED/122/SP24580/2018 (Magnetoencephalography resource facility) and Grant No: BT/PR20367/MED/122/22/2016 (Role of kynurenic acid, a glutamate receptor inhibitor, in hyperexcitability associated with mesial temporal lobe epilepsy), Department of Biotechnology, Ministry of Science \& Technology, Government. of India.

\section{Competing interests:}

The authors declare no competing interests.

\section{Declaration of transparency and scientific rigour:}

This Declaration acknowledges that this paper adheres to the principles for transparent reporting and scientific rigour of preclinical research as stated in the BJP guidelines for Design \& Analysis, and as recommended by funding agencies, publishers and other organisations engaged with supporting research.

\section{Abstract}

\section{Background and Purpose}

Glutamate receptor mediated enhanced excitatory neurotransmission is typically associated with mesial temporal lobe epilepsy with hippocampal sclerosis (MTLE-HS). Kynurenic acid (KYNA) and quinolinic 
acid (QUIN) are two important tryptophan-kynurenine pathway (KP) metabolites that modulate glutamate receptor activity. This study was designed to test the hypothesis that alteration in metabolism of KP metabolites in the hippocampus of patients with MTLE-HS contributes to abnormal glutamatergic transmission.

\section{Experimental Approach}

TKP metabolites level were determined using HPLC and LC-MS/MS in the hippocampal samples of patients with MTLE-HS compared to autopsy and non-seizure control samples. mRNA and protein expression of TKP enzymes were determined by qPCR and western blot. Spontaneous glutamatergic activities were recorded from pyramidal neurons in presence of kynurenine (KYN) and KYNA using whole cell patch clamp.

\section{Key Results}

We observed significantly reduced KYNA and elevated QUIN levels in the hippocampal samples, while KYN level remains unaltered. Spontaneous glutamatergic activity in the hippocampal samples was higher compared to that in non-seizure controls. Treatment with kynurenine inhibited the glutamatergic activity in non-seizure control samples but not in case of the hippocampal samples. However, exogenously applied KYNA inhibited glutamatergic activity in both non-seizure control and hippocampal samples. We also observed reduced levels of enzyme kynurenine aminotransferase II and its co-factor pyridoxal phosphate in the hippocampal samples.

\section{Conclusion}

Our findings indicate that altered metabolism of TKP metabolites in hippocampus could contribute to hyperglutamatergic tone in patients with MTLE-HS.

\section{Key Words:}

Mesial temporal lobe epilepsy, Hippocampal sclerosis, Kynurenic acid, Quinolinic acid,

Hyperexcitability, kynurenine pathway

\section{List of abbreviations:}

MTLE-HS: Mesial temporal lobe epilepsy with hippocampal sclerosis; nAChR: nicotinic acetyl choline receptors; KP: tryptophan-kynurenine pathway; KYNA: kynurenic acid; KYN: L-kynurenine; TRP: Tryptophan; IDO: indoleamine 2,3- dioxygenase; KAT II: kynurenine aminotransferase; PLP: pyridoxal phosphate; PNPO: Pyridoxamine 5'-Phosphate Oxidase; QUIN: quinolinic acid; KMO: Kynurnine-3-monooxygenase; EPSCs: excitatory postsynaptic currents; IPSCs: inhibitory postsynaptic currents.

\section{Bullet point summary (15 words max)}

\section{What is already known}

Kynurenine pathway metabolites are closely associated with glutamatergic excitatory synaptic activity inside the brain.

\section{What this study adds}

- Modulation of key enzymes leads to altered levels of kynurenine metabolites in the MTLE-HS hippocampus.

- Altered kynurenine pathway metabolite levels contribute to generation of enhanced glutamatergic synaptic transmissions.

\section{Clinical significance}

- Tight association between concentration of hippocampal kynurenine pathway metabolites and excitatory synaptic transmission. 
- This will help understand regulation of glutamate receptor function in the hippocampus of MTLE-HS patients.

\section{Introduction}

Tryptophan-kynurenine pathway (KP) is the principal route for tryptophan (TRP) metabolism. Almost $95 \%$ of TRP is directed to KP, catabolised through several "kynurenines", and generates $\mathrm{NAD}^{+}$as end product (Schwarcz et al., 2012; Vécsei et al., 2013). Kynurenic acid is one of the most important neuroactive "kynurenines" which selectively inhibits glycine co-agonist site of NMDA receptors at low concentration and a broad-spectrum antagonist of all excitatory amino receptors at high micromolecular concentration (Perkins \& Stone, 1982; Kessler et al., 1989). KYNA is known to interact directly with NMDA receptors, which is shown to contribute to the maintenance of glutamatergic synaptic activity in pyramidal neurons of the hippocampus (Cull-Candy et al., 2001). The inhibition of glutamatergic activity by KYNA could also be attributed to its effect on presynaptic nicotinic acetylcholine receptors which may reduce glutamate release (Carpenedo et al., 2001). Upon entering into TKP, TRP converts into L-kynurenine (KYN) by indoleamine2,3-dioxygenase (IDO) (Moroni et al., 2012). From KYN, the pathway divided into two branches. One leads to irreversible transamination of KYN to KYNA by kynurenine aminotransferase II (KAT II) and its co-factor pyridoxal phosphate (PLP) which is synthesized from dietary vitamin $\mathrm{B}_{6}$ by Pyridoxamine 5'-Phosphate Oxidase (PNPO) (Moroni et al., 2012; Musayev et al., 2009). Through another branch, KYN is converted into quinolinic acid (QUIN) which is an agonist of NMDA receptor and kynurnine-3monooxygenase (KMO) is the rate-limiting enzyme for QUIN synthesis (Schwarcz et al., 2012). Under physiological condition QUIN/KYNA ratio is critically maintained, as any fluctuation may lead to severe neuropathological conditions (Schwarcz et al., 2012). Fluctuation in KYNA concentration within the brain is associated with changes in neurotransmitters concentration (Carpenedo et al., 2001; Rassoulpour et al., 2005), and electrophysiological manifestation of epilepsy in animal models (Kamiński et al., 2003; Szyndler et al., 2012; Maciejak et al., 2009). Reduction in the cerebrospinal fluid (CSF) KYNA level has been reported in pediatric epilepsy patients (Yamamoto et al., 1994, 1995). However, increase in the level of KYNA in mammalian brain or CSF is associated with reduction of excitatory neurotransmission (Schwarcz et al., 2012). Alteration of KYNA and QUIN in central nervous system may constitute a common occurrence of abnormal synaptic transmission which has a pivotal role in generation of epileptogenesis.

Mesial temporal lobe epilepsy with hippocampal sclerosis (MTLE-HS) is the most common substrate for drug resistant epilepsy (DRE) (Jallon et al., 2001; Jin et al., 2015). Although origin of seizure generation are diversified, one prevailing hypothesis for hyperexcitation is the imbalance between excitatory and inhibitory synaptic transmissions mediated by glutamate (NMDA, AMPA/kainate) and GABA receptors. Under resting state, augmented synaptic endogenous NMDA receptor tone (Banerjee et al., 2015), differential upregulation of NR1 (de Moura et al. 2012), NR2A and NR2B subunits of this receptor, glutamatergic network reorganisation and synchronised excitatory interconnections within hippocampus contribute towards hyperexcitation (Banerjee et al., 2017). $\alpha 7$ nicotinic receptors (nAChR) also contributes to enhanced hippocampal glutamatergic activity in MTLE-HS (Banerjee et al., 2020). Presently, surgical intervention which includes anterior temporal lobectomy and amygdalo-hippocampectomy is the only available therapeutic option to achieve seizure freedom (Josephson et al., 2013; Engel, 2001). This study was designed to test the central hypothesis that alteration in metabolism of KP pathway metabolites in the hippocampus of patients with MTLE-HS contributes to abnormal glutamatergic transmission.

\section{Methods}

\section{Patients}

Drug resistant MTLE-HS patients $(\mathrm{n}=55)$ underwent standard presurgical workups which include video electroencephalogram (vEEG), epilepsy protocol MRI, interictal fluoro-2-deoxyglucose positron emission tomography (FDG-PET), ictal single photon emissioncomputer tomography (SPECT) and magnetoencephalography (MEG) [22]. Patient details were discussed weekly at epilepsy surgery meeting attended by epileptologists, epilepsysurgeon, neuroradiologists and nuclear-medicine specialists where patients were selected for 
surgery based on concordance of above-mentioned diagnostic procedures. Surgical resection involves anterior temporal lobectomy with amygdalohippocampectomy. A portion of resected hippocampus was analysed by neuropathologist for confirmation of the diagnosis of MTLE-HS. All these patients were seizure-free postoperatively (class I Engel outcome) (Supplementary Table 1) (Engel, 1983). Resected hippocampal tissues from patients who underwent autopsies and devoid of any history of brain pathology were collected within $12 \mathrm{~h}$ of death and used as autopsy controls $(\mathrm{n}=15)$ (Supplementary Table 2). For experiments involving cellular electrophysiological studies resected cortical tissues obtained from surrounding areas of brain tumours during surgical resection, which was also a part of planned surgical resection, in patients with glioma without any history of seizures were considered as non-seizure controls $(\mathrm{n}=20)$ molecular as autopsy control samples were not suitable for these experiments (Supplementary Table 3) (Banerjee et al., 2015; 2017; 2020). Samples were collected from 2015 to 2019 at the All India Institute of Medical Sciences in New Delhi, which is the referral center for epilepsy surgery in northern India. All procedures performed in studies involving human participants were in accordance with the ethical standards of the institutional and/or national research committee and with the 1964 Helsinki Declaration and its later amendments or comparable ethical standards.

The study was ethically approved by the Institutional Ethics Committee, All India Institute of Medical Sciences, New Delhi, India (IECPG/-38/27.11.2015, RT-4/30.12.2015). Informed consent was obtained from all patients or their legally authorized representatives prior to inclusion in this study.

A small portion of resected tissue was stored for fixation in $0.1 \mathrm{M}$ phosphate buffer containing $4 \%$ paraformaldehyde, $\mathrm{pH} 7.4$ for 72 hours. Post fixation, the tissues were dehydrated, embedded and cut into $6 \mu \mathrm{M}$ thick sections with a microtome and collected on poly L-lysine coated slides. Haematoxylineosin stained sections were used for cytoarchitecture. Immunohistochemistry was performed using with anti-GFAP (1:1000; Cat\# ab7260, RRID: AB_305808), or with anti-NeuN (1:1000; Cat\# ab104225, RRID: AB_10711153) primary antibodies. Secondary antibody was Biotinylated Goat Anti-Rabbit IgG antibody (1:1000; Vector Laboratories Cat\# BA-1000, RRID: AB_2313606)

\section{High performance liquid chromatography (HPLC)}

Quantification of metabolites tryptophan (TRP), KYN, KYNA, and PLP was performed by HPLCfluorescence detection.

Sample preparation: Within 5 min of resection from the brain, the tissue samples were kept in $1.0 \mathrm{ml}$ of 0.1 $\mathrm{M}$ perchloric acid and stored at $-80^{\circ} \mathrm{C}$. The frozen tissues were thawed on ice, weighed and placed in $2 \mathrm{ml}$ cryotubes. The samples were homogenised in ten volume of methanol/water $(85: 15, \mathrm{v} / \mathrm{v})$, centrifuged for $15 \mathrm{~min}$ at $10,000 \mathrm{rpm}$ at $0^{\circ} \mathrm{C}$. The supernatant was removed and stored at $-80^{\circ} \mathrm{C}$ until the time of chromatographic analysis.

For HPLC-fluorescence detection, reverse phase isocratic HPLC was performed (Shimadzu Prominence). Separation of the metabolites was achieved with a C18 column (Phenomenex Luna; $250 \mathrm{~mm}$ length, 4.6 $\mathrm{mm}$ internal diameter, $100 \AA$ pose size and $5 \mu \mathrm{m}$ particle size). The column temperature was maintained at $37^{\circ} \mathrm{C}$. The mobile phase, pumped at a flow rate of $1 \mathrm{ml} / \mathrm{min}$, was consisted of $50 \mathrm{mM}$ acetic acid, 100 $\mathrm{mM}$ zinc acetate containing $3 \%$ acetonitrile $(\mathrm{pH} 4.7)$. Ten microliter sample volume was injected into the system through an autosampler. The detection system was a fluorescence detector (RF 20A, Shimadzu, Japan) with dual wavelength simultaneous monitoring capability. Excitation, emission wavelength spectra and retention time for TRP, KYN, KYNA, and PLP were $297 \mathrm{~nm} / 348 \mathrm{~nm}, 365 \mathrm{~nm} / 480 \mathrm{~nm}, 344 \mathrm{~nm} / 404 \mathrm{~nm}$ and 300nm/400nm (Xiao et al., 2008; Cabo et al., 2014).

\section{Liquid chromatography-coupled tandem mass spectroscopy (LC-MS/MS)}

Quantification of metabolites Quinolinic acid (QUIN), and neurotransmitters glutamate, GABA was performed by LC-MS/MS.

Sample preparation : Within the supernatant, which was used for HPLC, $100 \mu \mathrm{l}$ extraction solvent containing $0.1 \mathrm{M}$ formic acid was added and centrifuged at $2000 \mathrm{rpm}$ for $5 \mathrm{~min}$ at $0^{\circ} \mathrm{C}$. The supernatant was again 
collected and immediately used for LC-MS/MS. One hundred sixty microliter of each sample was loaded into a 96 well-plate and subjected for analysis using LC-MS/MS (Roy et al., 2014).

LC-MS/MS system consisted of an ultra-HPLC (Agilent 1290 II) coupled to an Applied Bio systems API 4000 QTRAP triple quadrupole mass spectrometer (ABSciex). HPLC separation was achieved with a Waters Acquity UPLC BEH C18 column $(50 \mathrm{~mm}$ length, $2.1 \mathrm{~mm}$ internal diameter, $130 \AA$ pose size and $1.7 \mu \mathrm{m}$ particle size). The column temperature was maintained at $25^{\circ} \mathrm{C}$. The mobile phase, pumped at $0.2 \mathrm{~mL} / \mathrm{min}$, was consisted of methanol (eluent A) and $0.05 \%(\mathrm{v} / \mathrm{v}$ ) formic acid in water with $1 \mathrm{mM}$ of heptafluorobutyric acid (eluent B). The chromatographic gradient run was optimized according to the separation of analyte peaks and their shape. The total chromatographic run time was 8 minutes for a single run. The gradient run was started with $5 \%$ of eluent A and was maintained for 1 minute at a flow rate of $0.2 \mathrm{ml} / \mathrm{min}$. After that it was shifted to $100 \%$ eluent A with a flow rate of $0.4 \mathrm{ml} / \mathrm{min}$ in next $1.5 \mathrm{~min}$. This condition was maintained for the next $3 \mathrm{~min}$ and after that first line condition was achieved in next $0.5 \mathrm{~min}$ and was maintained for next $2 \mathrm{~min}$. The mass spectrometer was operated in positive ionisation utilising an electro spray ionisation (ESI) source. GABA, Glu, QUIN standards (Sigma-Aldrich, MO, USA; 100ng/ml in water) were infused into mass spectrometer using a pump with microsyringe (Hamilton, Switzerland) at the rate of $5 \mu \mathrm{l} / \mathrm{min}$ to tune the MS/MS system at different transitions of those compounds. Compound and instrument dependent parameters were optimized to get the maximum ion intensity using the inbuilt algorithm of Analyst 4.1 software. The multiple reaction monitoring (MRM) transitions of 104/87, 104/58.1 were used for GABA, 148/130, 148/84.2 for glutamate, and 121.83/77.8, 121.83/93.9 were used for QUIN. Nitrogen was used for the collision in MRM.

\section{Whole-cell patch clamp Electrophysiology}

These experiments were performed as described earlier (Benerjee et al., 2017; 2020). Briefly, resected hippocampal and non-seizure control tumour periphery tissues were placed in ice-cold, carbogenated $\left(95 \% \mathrm{O}_{2}\right.$, $5 \% \mathrm{CO}_{2}$ ) artificial cerebrospinal fluid (ACSF; NaCl, $125 \mathrm{mM}$; KCl, $2.5 \mathrm{mM} ; \mathrm{CaCl}_{2}, 2 \mathrm{mM}$; $\mathrm{NaHCO}_{3}, 25$ $\mathrm{mM} ; \mathrm{NaH}_{2} \mathrm{PO}_{4}, 1.25 \mathrm{mM} ; \mathrm{MgCl}_{2}, 1 \mathrm{mM}$; and Glucose,25 mM.) and brought to the laboratory, followed by $350 \mu \mathrm{m}$ thick slices were prepared in a vibratome. Slices were prepared by making tangential cuts to the outer surface of the cortical specimens and were incubated at room temperature for 30 mins and transferred to a recording chamber which was perfused $(2 \mathrm{ml} / \mathrm{min})$ with carbogenated ACSF. Normal appearing pyramidal neurons with thick pyramid like soma and single tapering dendrite were morphologically identified visually using Infrared-assisted video-microscopy with differential interference contrast (IR-DIC). Cells on the surface slice preparations were usually dead, so we used the pale-looking pyramidal neurons from layer III or IV for our studies. Patch pipettes with resistance of 3-5 M $\Omega$ were filled with internal solution containing HEPES, $10 \mathrm{mM} ; \mathrm{MgCl}_{2}, 2 \mathrm{mM}$, Cs-methanesulfonate, $130 \mathrm{mM}$; EGTA, $10 \mathrm{mM}$; CsCl, $10 \mathrm{mM}$. Passive membrane properties of neurons were determined by "membrane test" function of pCLAMP 10.0 software (Molecular Devices, USA). Whole cell patch clamp recordings were performed from those pyramidal neurons using an amplifier (Axopatch 200B, Molecular Devices, USA). Spontaneous excitatory postsynaptic currents (EPSCs) were recorded at $-70 \mathrm{mV}$ holding potential and spontaneous inhibitory postsynaptic currents (IPSCs) at $0 \mathrm{mV}$ respectively. The access resistance were between 15-20 M $\Omega$ and change of more than $20 \%$ from this range was not reliable and data of those neurons were discarded. The leak current during recording was between 50-150 pA. If it was more than $200 \mathrm{pA}$, the data became unreliable. All recordings were performed at room temperature $\left(22-24^{\circ} \mathrm{C}\right)$

Data were analysed in pCLAMP 10.0 software. Frequency, amplitude, rise time (10-90\%) and decay time constant $\left(\tau_{d}\right)$ of the sEPSCs and sIPSCs were measured. The amplitude threshold for sEPSCs and sIPSCs were $-5 \mathrm{pA}$ and $+10 \mathrm{pA}$ respectively. All recordings were visually inspected to select events which show a steep rising and exponential decay phase for kinetic analysis of EPSCs and IPSCs. Events that did not show the above-mentioned rise and decay phase were not typical synaptic waveform and these were rejected manually. Events that showed multiple peaks were excluded for kinetic analysis (rise time and decay time constant) but included for frequency calculation as multiple events. Cumulative distribution of inter-event interval and amplitude in non-seizure control vs MTLE-HS groups were compared using Kolmogorov-Smirnov test (K-S 
test).

\section{mRNA expression analysis}

Quantitative real time PCR (qPCR) was performed to estimate mRNA level expression of IDO, KAT II, KMO, PNPO enzymes. HPRT (hypoxanthine phosphoribosyl-transferase) was used as house-keeping gene. All experiments were performed in triplicates. Specified primers for all the genes were designed using Primer3 and BLAST (Primer3 Input version 0.4.0 and BLAST; http://www.ncbi.nlm.nih.gov/tools/primer-blast/). For each target gene, primers were designed to target all possible transcript variants mentioned in the reference sequence (RefSeq) database (http://www.ncbi.nlm.nih.gov/refseq). RNAs were extracted using Trizol reagent (Qiagen RNeasy kit); quantified using Qubit 3 fluorometer (Thermo scientific) and Qubit RNA BR assay kit. Reverse transcription was performed to create cDNA using high capacity cDNA reverse transcription kit (Thermo scientific) according to the manufacturer's protocol. qPCR was performed with cDNA, suitable primers using SsoFast Eva Green Supermix (Bio-Rad) on a CFX-96 real time system (BioRad). The cycle parameter was: 5 mins at $95^{\circ} \mathrm{C}$ for initial hot-start, followed by 40 cycles of 15 secs at $95^{\circ} \mathrm{C}$ for denaturation and 30secs at $60^{\circ} \mathrm{C}$ for annealing and extension. Specificity of the expected PCR products were verified using melting-curve analysis. $\mathrm{C}_{\mathrm{T}}$ values (cycle threshold) were obtained for individual samples and delta $C_{\mathrm{T}}\left(\Delta \mathrm{C}_{\mathrm{T}}\right)$ were calculated by subtracting the $\mathrm{C}_{\mathrm{T}}$ values of house-keeping gene (HPRT) from that of the gene of interests. These $\Delta \mathrm{C}_{\mathrm{T}}$ values were used to compare extent of the mRNA expression between the two groups where mRNA expression was inversely proportional to the $\Delta \mathrm{C}_{\mathrm{T}}$ value (Livak \& Schmittgen, 2001). The primer sequences were: for HPRT-F, 5'-GCTTTCCTTGGTCAGGCAGTA-3', for HPRT-R, 5'-GGTCCTTTTCACCAGCAAGCT-3', for IDO-F, 5'-GCGCTGTTGGAAATAGCTTC-3', for IDO-R, 5'TGATCGTGGATTTGGTGAAA-3', for KATII-F, 5'-TTAATGCTCCCTGGAAATGC-3', for KATII-R, 5'GAAGGCCACATCCATCTGTT-3', for KMO-F, 5'-CACTGTGTACTGCTGGGAGA-3', for KMO-R, 5'ATCGCGTGATCATCTGGGAT-3', for PNPO-F, 5'-ACCCAGGCCCTTCTTTCTAA-3', and for PNPO-R, 5'-TACGCCACCATTACACTCCA-3'.

\section{Protein expression analysis}

Protein isolation: For total protein extraction, hippocampal and cortical samples were homogenized in RIPA buffer (150 mM NaCl, 20 mM Tris-HCl, 1mM EGTA, 1mM Na ${ }_{2}$ EDTA, 1\%sodium deoxycholate, 1\% NP-40, $\mathrm{pH}$ 7.4) supplemented with $1 \%$ protease inhibitor cocktail, $1 \mathrm{mM}$ phenylmethylsulfonyl fluoride (PMSF), $10 \mathrm{mM}$ sodium fluoride $(\mathrm{NaF})$, and $2 \mathrm{mM}$ sodium orthovanadate $\left(\mathrm{Na}_{3} \mathrm{VO}_{4}\right)$ (Sigma aldrich). The samples were centrifuged twice for $15 \mathrm{~min}$ each at $15,000 \mathrm{rpm}$ at $0^{\circ} \mathrm{C}$. The supernatant was removed and the protein concentration was determined using Bicinchoninic Acid Assay (BCA) kit (Pierce) and a microplate reader at $562 \mathrm{~nm}$ (Bio-Rad).

Western blot : The immuno-related procedures used comply with the recommendations made by the British Journal of Pharmacology (Alexander et al., 2018). Equal amount of proteins was loaded along with dual colour ladder (Bio-Rad) on 12-15\% polyacrylamide gels and transferred onto Polyvinylidene fluoride (PVDF) membranes. Membranes were blocked with $3 \%$ bovine serum albumin in phosphate buffered saline with $0.1 \%$ tween-20 for $2 \mathrm{hrs}$ at room temperature; followed by primary antibodies incubation including human antiKAT II (1:700; abcam Cat\# ab83918, RRID: AB_2219600), human anti-KMO (1:700; abcam Cat\# ab130959, RRID: AB_11156090), human anti-PNPO (1:1000; Cat\# ab83875, RRID: AB_1861751) diluted in phosphate buffered saline with $0.1 \%$ tween- 20 for $4 \mathrm{oC}$ overnight and subsequently HRP conjugated secondary antibodies incubation (1:1000, Santa Cruz Cat\# sc-2004, RRID: AB_631746) diluted in same buffer for 2 hrs at room temperature. Chemiluminescence signals were generated using Super Signal West Pico Chemiluminescent Substrate (Thermo Scientific) and images were captured on a Bio-Rad Chemi Doc MP system. Band intensities were quantified by ImageJ software. Protein expressions were normalised with respect to loading control glyceraldehyde-3-phosphate dehydrogenase (GAPDH).

ELISA: Protein expression of Indoleamine 2,3-dioxygenase (IDO) enzyme was performed by ELISA using commercially available kits (Catalogue number: DY6030-05, R \& D Systems, USA) according to manufacturers' instruction. The experiment was performed in triplicates. Optical density was determined in 
a multimode reader (Synergy HTX, Biotek, USA) at $450 \mathrm{~nm}$ and wavelength was corrected as $540 \mathrm{~nm}$ (Nurnberger et al., 2019).

\section{Group size, Data and Statistical analysis}

There is no prior study regarding the role of kynurenine pathway metabolites on glutamate receptor mediated neurotransmission in MTLE-HS patients. In the present study, it is not possible to calculate the sample size as there is no prior reports. It is well established that $30 \%$ of patients with MTLE-HS are drug resistant (Kwan et al., 2010). Based on this fact, we have collected resected hippocampal samples from 55 drug resistant MTLE-HS patients underwent surgical intervention from seizure freedom over a period of 4 years from 2015 to 2019. Here we have used $n=6$ as minimum number of repetitions required to have $>95 \%$ possibility $(\mathrm{p}<0.05)$ to detect. This sample size satisfies the guidelines of the British Journal of Pharmacology for preclinical studies, where $\mathrm{n}$ refers to independent values and not replicates (available at: https://bpspubs.onlinelibrary.wiley.com/hub/journal/14765381/author-guidelines.html).

We have complied with the recommendations of the British Journal of Pharmacology on experimental design and analysis in pharmacology (Curtis et al., 2018). Statistical analyses were performed on Sigma Plot 13.0 (RRID: SCR_003210) and GraphPad Prism 6.0 software (RRID: SCR_002798). When normal distribution and equal-variance were valid, statistical significance between two groups were evaluated by two tailed unpaired t-test otherwise Mann Whitney test was used for evaluation and between more than two groups by Kruskal-Wallis non-parametric ANOVA followed by multiple comparison with Dunn's test was performed. Cumulative distribution of electrophysiological data was analysed by Kolmogorov-Smirnov test. The correlation between duration of seizure and concentration of metabolites was performed using Spearman's rank correlation test. The least squares method was used to plot the best fit line and to predict the behaviour of dependent variables (KYNA, PLP, and QUIN). The goodness of fit was calculated through $\mathrm{R}^{2}$. The overall significance ( $\mathrm{P}$ value) was calculated by F-test. Data were presented as a mean +- SEM. $\mathrm{P}<0.05$ was considered significant.

\section{Materials}

All standard compounds for HPLC and LC-MS/MS as well as all reagents for patch clamp were purchased from Sigma Aldrich (St. Louis, MO, USA). All primary antibodies in western blot experiment were purchased from Abcam (Cambridge, UK) and secondary antibody was purchased from Santa Cruz (USA).

\section{Results}

\section{Histopathological examinations revealed evidence of hippocampal sclerosis}

Haematoxylin-Eosin staining of the hippocampal sections obtained from patients with MTLE-HS revealed typical sclerotic hippocampus consists of massive cell loss, cystic cavities, neuronal degeneration, gliosis and infiltration of inflammatory cells (Figure 1a). The NeuN immunohistochemistry confirms pyramidal neuron loss, presence of ischemic neurons and abnormal neuronal architecture (Figure 1b). Another hallmark of the hippocampal sclerosis is reactive astrogliosis with hypertrophic astrocytes manifesting distinctive GFAP staining and thick, long projections (Figure 1c). In autopsy control tissue sections, negligible neuronal loss was observed (Figure 1d and e). The GFAP immunohistochemistry did not reveal any reactive astrogliosis (Figure 1f). These data suggested that architectural alterations like, pyramidal cell apoptosis, necrosis and reactive astrogliosis were observed in the hippocampal samples of the MTLE-HS patients.

\section{Tryptophan-kynurenine pathway metabolite levels were altered in MTLE-HS}

We have also quantified the TKP metabolites namely TRP, KYN, KYNA, QUIN and neurotransmitters glutamate and GABA in tissues obtained from the surgically resected hippocampal samples obtained from patients with MTLE-HS and compared it with autopsy control hippocampus tissues. The concentration of TRP was significantly reduced (Figure 2a) in the hippocampal samples. Although KYN concentration was not significantly altered (Figure 2b), but that of KYNA was significantly reduced in the hippocampal samples (Figure 2d). The tryptophan kynurenine ratio was also significantly reduced in the hippocampal 
samples (Figure 2c). The concentration of QUIN was significantly enhanced in the hippocampal samples (Figure 2e). The ratio of QUIN and KYNA was also significantly enhanced in the hippocampal samples (Figure 2f). The concentration of glutamate (Figure 2g), GABA (Figure 2h) and glutamate GABA ratio (Figure 2i), neither of those was significantly altered between the two groups.

To investigate the de novo synthesis pattern of KYNA and QUIN in tissues, $300 \mu \mathrm{M}$ thick slices were prepared from the surgically resected hippocampus tissues and tumour periphery non-seizure control tissues, incubated in ACSF (artificial cerebrospinal fluid) containing $200 \mu \mathrm{M} \mathrm{KYN} \mathrm{(saturating} \mathrm{concentration),} \mathrm{precursor} \mathrm{of}$ both KYNA and QUIN, and incubated at $30^{\circ} \mathrm{C}$ in a water bath for 2 hours (Turski et al., 1989). At the end of the incubation period, the ACSF was aliquoted; KYNA and QUIN were estimated by HPLC and LC-MS/MS respectively. We observed a similar pattern of results as earlier. The de novo synthesis of KYNA was significantly reduced in the hippocampal samples obtained from patients with MTLE-HS (Figure 2j). Similarly, the de novo synthesis of QUIN was significantly enhanced in the hippocampal samples obtained from patients with MTLE-HS (Figure 2k).

Enhanced spontaneous glutamatergic activity in the hippocampal samples obtained from patients with MTLE-HS

For all the electrophysiological experiments, we have used tumour periphery non-seizure control tissues for comparing with hippocampal samples obtained from patients with MTLE-HS. Passive membrane properties of pyramidal neurons in slice preparation obtained from hippocampal samples of patients with MTLE-HS and that of tumour periphery non-seizure controls were comparable (Cell capacitance $163 \pm 16 \mathrm{pF}$ in nonseizure control vs $171 \pm 21 \mathrm{pF}$ in MTLE-HS; input resistance $148 \pm 23 \mathrm{M} \Omega$ in non-seizure control vs $157 \pm$ $27 \mathrm{M} \Omega$ in MTLE-HS). We recorded spontaneous excitatory postsynaptic currents (EPSCs) from pyramidal neurons at $-70 \mathrm{mV}$ holding potential under whole-cell voltage clamp configuration in $300 \mu \mathrm{M}$ thick slice preparations obtained from the MTLE-HS and non-seizure control groups. These glutamatergic events were inward currents which were completely abolished following 10 minutes bath perfusion of the slices with ACSF containing NMDA receptor antagonist APV $(50 \mu \mathrm{M})$ and AMPA receptor antagonist CNQX $(10 \mu \mathrm{M})$, implying that these events were mediated by both NMDA and AMPA/kainate receptors (Figure 3a). These events were unaffected by bath perfusion with $\mathrm{GABA}_{\mathrm{A}}$ receptor antagonist bicuculline $(10 \mu \mathrm{M})$, indicating that the resting state spontaneous glutamatergic activity was independent of GABAergic influence, in these slice preparations. The frequency and amplitude of the spontaneous EPSCs were significantly increased in the hippocampal samples (Table 1). The normalised cumulative distribution of inter-event intervals shifted significantly to left side, whereas that of peak amplitudes shifted significantly to right side in the hippocampal samples (Figure $3 \mathrm{~b}$ and $\mathrm{c}$ ). However, Rise time and $\tau_{\mathrm{d}}$ was not affected (Table 1 ). These findings suggested that resting state glutamatergic activity was enhanced in the hippocampal samples of the MTLE-HS patients.

We recorded the spontaneous inhibitory postsynaptic currents (IPSCs) at $0 \mathrm{mV}$ from the pyramidal neurons in the slice preparations of resected brain specimens obtained from patients with MTLE-HS and non-seizure controls. The spontaneous IPSCs were completely blocked following 15 minutes bath perfusion of those slices with ACSF containing bicuculline $(10 \mu \mathrm{M}$; Figure 3d). However, we did not observe any significant alteration in the frequency as well as amplitude of IPSCs between the two groups (Table 1). The cumulative distribution of inter-event intervals as well as peak amplitudes remain unchanged (Figure 3e and f). Then we calculated the ratio of frequency and amplitude of glutamatergic-GABAergic activities in a cohort of neurons where EPSCs and IPSCs were recorded from the same neuron. We found that the ratio of frequency as well as amplitude were significantly increased in the hippocampal samples (control, $\mathrm{n}=5$; MTLE-HS, $\mathrm{n}=5$; frequency ratio $0.39 \pm 0.015$ in non-seizure control vs $0.62 \pm 0.010$ in MTLE-HS; amplitude ratio $0.53 \pm$ 0.014 in non-seizure control vs $0.71 \pm 0.21$ in MTLE-HS; Figure $3 \mathrm{~g}$ ).

\section{Reduced endogenous synthesis of KYNA may cause enhanced glutamatergic activity in MTLE- HS}

To investigate the role of the endogenously synthesized KYNA on glutamatergic neurotransmission, the tissue slices obtained from patients with MTLE-HS and non-seizure controls were incubated in well carbogenated 
$\left(95 \% \mathrm{O}_{2}+5 \% \mathrm{CO}_{2}\right)$ ACSF containing $200 \mu \mathrm{M} \mathrm{KYN} \mathrm{(precursor} \mathrm{for} \mathrm{KYNA)} \mathrm{for} \mathrm{around} 60$ mins and then EPSCs were recorded from the pyramidal neurons using the same solution as perfusate for 25 mins (total 85 mins). The reason behind the incubation with KYN was that, it will provide adequate time to synthesize sufficient amount of KYNA which will suppress the glutamatergic events. In the non-seizure control group $(\mathrm{n}=6)$, frequency and amplitude of the spontaneous EPSCs were significantly reduced after incubation with $200 \mu \mathrm{M}$ KYN in comparison to those of basal values (Table 1). The cumulative distribution of inter-event intervals displaced toward longer interval while that of peak amplitudes displaced toward shorter amplitude (Figure $4 \mathrm{~b}$ and $\mathrm{c}$ ). Rise time and $\tau_{\mathrm{d}}$ was not affected (Table 1). In case of the hippocampal samples of the MTLE-HS patients $(\mathrm{n}=16)$, the frequency as well as the amplitude of the spontaneous EPSCs were not significantly altered after incubation with $200 \mu \mathrm{M}$ KYN in comparison to those of basal values (Table 1). The cumulative distribution of inter-event intervals as well as peak amplitudes remain unchanged (Figure 4e and $\mathrm{f}$ ). Rise time and $\tau_{d}$ was not affected (Table 1). The magnitude of the percentage reduction of the frequency was significantly reduced in the hippocampal samples in comparison to the non-epileptic controls $(51.9 \pm 1.56 \%$ in non-epileptic control vs $4.6 \pm 0.84 \%$ in MTLE-HS; Figure $4 \mathrm{~g})$. This result was consistent with above mentioned results that in the hippocampal samples of the MTLE-HS patients, sufficient amount of KYNA is not synthesized from its precursor KYN de novo and consequently enhanced glutamatergic activity was observed.

\section{Exogenously applied KYNA suppressed spontaneous glutamatergic activity in the hippocam- pal samples obtained from patients with MTLE-HS}

To investigate the role of exogenously applied KYNA on glutamatergic neurotransmission, the tissue slices of the above-mentioned groups were perfused with $10 \mu \mathrm{M}$ KYNA for 30 mins. The frequency and amplitude were significantly reduced after 30 mins perfusion of KYNA in comparison to those of basal values in both groups (Table 1). The cumulative distribution of inter-event intervals displaced toward longer interval and that of peak amplitudes displaced towards shorter amplitude (Figure $5 \mathrm{~b}, \mathrm{c}$ and e, f). However, Rise time and $\tau_{\mathrm{d}}$ was not affected (Table 1). The magnitude of percentage reduction of the frequency was significantly enhanced in the hippocampal samples in comparison to the non-seizure controls $(16.34 \pm 0.92 \%$ in non-seizure control vs $30.72 \pm 1.87 \%$ in MTLE-HS; Figure $5 \mathrm{~g}$ ). At low micro molecular concentration, KYNA predominantly exerts inhibition through NMDA receptors; but in the present scenario, inhibitory action of KYNA was significantly enhanced in case of MTLE-HS which may be due to upregulation of NMDA receptors. Now, to investigate the contribution of inhibitory action of KYNA mediated through the NMDA receptors, the slices were perfused with 50uM APV alone for 10 mins which blocked only NMDA receptors. APV suppressed the frequency by $42.83 \pm 2.26 \%$ in the non-seizure controls and $58.17 \pm 2.06 \%$ in the MTLE-HS group respectively (Figure $5 \mathrm{~h}$ and i) with a significant difference between the two groups suggesting the role of enhanced tonic NMDA receptor activity in altered synaptic transmission in the hippocampal samples of the MTLE-HS patients. For KYNA, the magnitude of reduction of the frequency was significantly reduced than that of APV (Figure 5j), suggesting that although its primary target are NMDA receptors, but it also has some target sites which are different from it.

\section{Reduced endogenous KYNA synthesis was due to altered levels of KAT II and PLP}

From the above experiments, it was evident that endogenous KYNA synthesis was reduced and QUIN synthesis was elevated in the hippocampal samples obtained from patients with MTLE-HS which could be attributed to the dysfunctional machinery responsible for synthesis of those. KYNA is synthesized from KYN in presence of enzyme KAT II. To address whether the altered expression of this enzyme was correlated with the reduced synthesis of its product, we investigated its mRNA and protein expression by quantitative reverse transcriptase polymerase chain reaction $(\mathrm{qPCR})$ and western blotting respectively. We found no alteration of mRNA expression in the hippocampal samples (Figure 6a). However, protein expression was significantly downregulated in the hippocampal samples (Figure 6b). However, the magnitude of this reduction was not proportional to that of reduction in concentration of KYNA. Given the fact that function of KAT II depends on the co-factor PLP, we estimated its concentration by HPLC and found that the concentration of PLP was significantly reduced in the hippocampal samples (Figure $6 \mathrm{~g}$ ). Then we investigated mRNA and 
protein expression of the enzyme PNPO which synthesizes PLP from pyridoxine. We found that both mRNA (Figure 6h) as well as protein expression of this enzyme (Figure 6i) were significantly downregulated in the hippocampal samples.

We investigated the expression profile of IDO, the rate-limiting enzyme for TRP to KYN conversion. Both mRNA (Figure 6c) as well as protein level expression (Figure 6d) were significantly upregulated in the hippocampal samples. We focused on the rate-limiting enzyme for QUIN synthesis which is KMO because as per our results, concentration of QUIN was higher in the hippocampal samples. Both mRNA (Figure 6e) as well as protein expression (Figure 6f) were not significantly altered in the hippocampal samples.

The concentration of metabolites in the hippocampal samples was correlated with seizure duration of patients with MTLE-HS

To investigate whether there was any correlation between the concentration of TKP metabolites (KYNA and its de novo synthesis, QUIN and its de novo synthesis, PLP) and total duration of seizure (duration between age of seizure onset and age of surgery as all patients achieve seizure freedom of Engel class I following surgery), we examined the concentration of each metabolite and it's de novosynthesis of each patient and correlated it with duration of seizure using non-parametric Spearman's correlation test. We observed that the concentration of KYNA (Figure 7a) and its de novo synthesis (Figure 7b) were inversely correlated with the duration of seizure. The concentration of PLP was also inversely correlated with the duration of seizure (Figure 7e). Although the concentration of QUIN was correlated with the duration of seizure (Figure 7c) but we could not observe any correlation between de novo synthesis of QUIN and the duration of seizure (Figure $7 \mathrm{~d}$ ).

\section{Discussion}

The present results demonstrate that (i) endogenous synthesis of KYNA, a glutamate receptor inhibitor, was lowered in the hippocampal samples obtained from patients with MTLE-HS, possibly due to reduced levels of the enzyme KAT II and its co-factor PLP. (ii) Reduced endogenous KYNA levels may contribute to the maintenance of enhanced synaptic glutamatergic activity on to the pyramidal neurons in slice preparations of the resected hippocampal samples obtained from patients with MTLE-HS. (iii) Under resting conditions, the effect of the reduced endogenous KYNA levels could be attributed to both pre- as well as post-synaptic regulation of glutamatergic activity. (iv) Endogenous synthesis of QUIN was elevated in the hippocampal samples obtained from patients with MTLE-HS. These concepts can help explain the potential contribution of TKP metabolites to hyperexcitability associated with MTLE-HS.

Reduction in endogenous KYNA synthesis contributes to the hyperexcitability in MTLE-HS

Astrocyte-neuron interaction play a crucial role in the regulation of glutamatergic activity. One of the regulation mechanisms is the conversion of KYN to KYNA in astrocytes which inhibits glutamate receptormediated synaptic transmission on to neurons (Moroni et al., 2012). Pyridoxine and PNPO has long history with epilepsy treatment (Watanabe, 1995; Ito et al., 2000; Kuo and Wang, 2002; Jaeger et al., 2016). We observed that the mRNA and protein expression of the PNPO enzyme were significantly downregulated in MTLE-HS patients leading to reduced PLP synthesis (Figure $7 \mathrm{~g}, \mathrm{~h}, \mathrm{i}$ ). Taken together, reduction in both KAT II expression as well as its co-factor PLP might be affecting de novo synthesis of KYNA from KYN. Our

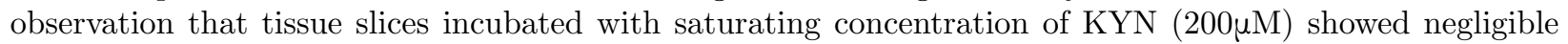
change in the frequency and amplitude of spontaneous EPSCs in the hippocampal samples of patients with MTLE-HS compared to that in non-seizure control samples(Figure 4e) can be attributed to both presynaptic as well as postsynaptic glutamatergic activity. KYNA inhibits action potential dependent spontaneous glutamatergic activities (Banerjee et al., 2012) and reduction of endogenous KYNA synthesis enhances hippocampal extracellular glutamate release as evidenced in animal models (Pocivavsek et al., 2011; Potter et al., 2010). Further experiments involving isolation of miniature EPSCs in the presence of KYNA may further help us understand the regulation of glutamatergic activity by quantal currents in the hippocampal samples obtained from patients with MTLE-HS. We suspect that the amount of KYNA synthesized de novo in the hippocampal samples was less than IC50 value of KYNA for $\alpha 7 \mathrm{nAChR}(1-8 \mu \mathrm{M})$ (Hilmas et al., 
2001) and glycine site of NMDA receptor (10-30 $\mu \mathrm{M})$ (Parsons et al., 1997). Consequently, presynaptic $\alpha 7$ $\mathrm{nAChR}$ dependent glutamate release was not regulated and action potential dependent presynaptic inputs were enhanced, leading to increase in the frequency of sEPSCs (Banerjee et al., 2020). The postsynaptic NMDA receptors were upregulated and functionally hyperactive in MTLE-HS hippocampus (Banerjee et al., 2017). As, postsynaptic glycine sites of NMDA receptors were also not competitively inhibited by KYNA, the amplitude of spontaneous EPSCs was also increased. Future studies involving incubating the brain slices with either the KAT II or PLP or PLP synthesizing enzyme PNPO will be beneficial to prove the central hypothesis more accurately by assessing the extent of recovery of KYNA synthesis in MTLE-HS.

We could not see significant alterations in GABAergic activities under resting state. This suggests that, under resting conditions, hyperexcitatory activities were primarily mediated by glutamate receptors in the hippocampal samples. Moreover, these MTLE-HS patients were on AEDs (Supplementary table 1), most of which are $\mathrm{GABA}_{\mathrm{A}}$ receptor modulators, may have compensated for the altered GABAergic activity in the hippocampal samples.

\section{Endogenous QUIN synthesis was elevated}

Our findings suggested increased expression and functional activity of IDO enzyme in the hippocampal samples (Figure 6c and d). Reduction in TRP/KYN ratio (Figure 2c) is suggestive of increased functional activity of IDO in those samples. We observed that with increase in duration of seizure, concentration of PLP was gradually decreases due to downregulation of PNPO. Consequently, due to less availability of PLP and downregulation of KAT II, concentration of KYNA gradually decreases with increase in duration of seizure. As synthesis of KYNA decreases, availability of substrate (KYN) increases with time. It is possible that due to this reason, in spite of unaltered expression of KMO, concentration of QUIN gradually increases with increase in duration of seizure. This suggests that in patients with MTLE-HS, increase in duration of seizures may redirect the kynurenine pathway towards reduced KYNA synthesis and increased QUIN synthesis which could contribute to enhanced glutamatergic synaptic transmission. An increase in QUIN/KYNA ratio causes hyperexcitation, excitotoxicity, astrogliosis and decrease in neuronal viability as evident from our histopathological findings (Figure 1a, b, c). Under physiological condition, astrocytes contain enzymes for degradation of QUIN, but during brain inflammation reactive astrocytes produce KYN which translocate to resident activated microglia or infiltrating macrophages to serve as substrate for QUIN synthesis (Guillemin et al., 2001). It has been shown that infusion of KYN in the pyriform cortex of chronic epileptic rats corresponds to progressive decline in extracellular KYNA concentration after achieving peak concentration, which is speculated to be due to downregulation of KAT in reactive astrocytes (Wu et al., 1991). In the present study, it is possible that reactive astrocytes/astrogliosis in the hippocampal samples (Figure 1C) not only associate with downregulation of KAT II enzyme (Figure 6a and b) but also facilitate synthesis of QUIN from microglia/macrophages in lieu of KYNA which ultimately contribute to glutamate receptor mediated hyperexcitability.

\section{Clinical significance of altered kynurenine metabolism and enhanced glutamatergic activity in MTLE-HS}

As KYNA modulates the hippocampal extracellular glutamate release through $\alpha 7 \mathrm{nAChR}$ (Carpenedo et al., 2001), it is possible that exogenously applied KYNA prevented presynaptic action potential-dependent glutamate release causing reduction in frequency of spontaneous EPSCs recorded from pyramidal neurons in resected hippocampal samples (Fig. 5E). As KYNA competitively inhibits postsynaptic glycine binding site of the NMDA receptors and NMDA receptor levels are high in patients with MTLE-HS, exogenous application of KYNA may increase its binding to postsynaptic NMDA receptors causing reduction in amplitude of spontaneous EPSCs (Figure 5e). In addition, we also observed that the magnitude of reduction of frequency of spontaneous EPSCs by KYNA in MTLE-HS was significantly less than that caused by specific NMDA receptor antagonist, APV (Figure 5i). This might suggests that, besides NMDA receptors, KYNA may have some other targets like GPR35 (Alkondon et al., 2015), aryl hydrocarbon receptors (DiNatale et al., 2010) or some other unknown sites; but their role in MTLE-HS is not known and needs further investigations. Moreover, future experiments involving comparison between KYNA and APV co-perfusion and KYNA or 
APV alone can further help to understand the presence of a cumulative or occlusive effect of these antagonists on NMDA receptor activity. Increase in endogenous QUIN level in hippocampal samples also contributes to glutamate receptor mediated hyperexcitability. QUIN binds with postsynaptic NMDA receptors which are upregulated and render those functionally hyperactive. It is tempting to speculate that decreased KYNA levels as well as increased QUIN levels in the hippocampus and hyperglutamatergic tone may contribute to seizure generation in patients with MTLE-HS.

The primary limitation for this human study is use of tumour periphery samples obtained from patients with low-grade gliomas as non-seizure control, as ethically it was not possible to get healthy hippocampal samples. The cell type variation, cell-specific expression pattern and subtype differences of glutamate receptors between the MTLE-HS samples and non-seizure control samples could also contribute to the changes observed in the frequency and amplitude of spontaneous EPSCs. Although previous studies have used samples obtained from non-MTLE patients for comparison with MTLE-HS samples, we believe that epileptic samples obtained from patients with non-MTLE (but DRE) could also have their own limitations as "control" as those tissues will also possess, the epilepsy related abnormalities which may significantly affect the comparative results of synaptic transmission between the two groups. In addition, it was not possible to obtain age and gender matched non-seizure control specimens for this study. All the patients recruited in this study were on a combination of anti-epileptic drugs (supplementary table 1), so the effect of these drugs on glutamatergic/GABAergic activity recorded from the pyramidal neurons of resected hippocampal samples cannot be ruled out.

\section{Conclusions}

Our findings suggest tight association between concentration of hippocampal kynurenine pathway metabolites and excitatory synaptic transmission and duration of seizure in patients with MTLE-HS. This will help understand regulation of glutamate receptor function in the hippocampus of patients with MTLE-HS.

\section{References}

Alkondon M, Pereira EF, Todd SW, Randall WR, Lane MV, Albuquerque EX (2015) Functional G-proteincoupled receptor 35 is expressed by neurons in the CA1 field of the hippocampus. Biochem Pharmacol. 93:506-518. Banerjee J, Alkondon M, Albuquerque EX (2012) Kynurenic acid inhibits glutamatergic transmission to CA1 pyramidal neurons via a7 nAChR-dependent and -independent mechanisms. Biochem Pharmacol. 84:1078-1087. Banerjee J, Banerjee Dixit A, Srivastava A, Ramanujam B, Kakkar A, Sarkar C, Tripathi M, Chandra PS (2017) Altered glutamatergic tone reveals two distinct resting state networks at the cellular level in hippocampal sclerosis. Sci Rep. 7:319 Banerjee J, Banerjee Dixit A, Tripathi M, Sarkar C, Gupta YK, Chandra PS (2015) Enhanced endogenous activation of NMDA receptors in pyramidal neurons of hippocampal tissues from patients with mesial temporal lobe epilepsy: A mechanism of hyper excitation. Epilepsy Res. 117:11-16. Banerjee J, Dey S, Banerjee Dixit A, Tripathi M, Doddamani R, Sharma MC, Chandra PS (2020) $\alpha 7$ nicotinic receptors contributes to glutamatergic activity in the hippocampus of patients with mesial temporal lobe epilepsy with hippocampal sclerosis (MTLE-HS). J Neural Transm. 127:14411446. Cabo R, Kozik K, Milanowski M, Hernes S, Slettan A, Haugen M, Ye S, Blomhoff R, Mansoor MA (2014) A simple high-performance liquid chromatography (HPLC) method for the measurement of pyridoxal5-phosphate and 4-pyridoxic acid in human plasma. Clin Chim Acta. 433:150-156 Carpenedo R, Pittaluga A, Cozzi A, Attucci S, Galli A, Raiteriet M, Moroni F (2001) Presynaptic kynurenate-sensitive receptors inhibit glutamate release. Eur J Neurosci. 13:2141-2147. de Moura, Tirapelli DP, Neder L, Saggioro FP, Sakamoto AC, Velasco TR, Panepucci RA, Leite JP, Assirati Júnior JA, Colli BO, Carlotti Júnior CG (2012) Amygdala gene expression of NMDA and GABA(A) receptors in patients with mesial temporal lobe epilepsy. Hippocampus. 22:92-97 DiNatale BC, Murray IA, Schroeder JC, Flaveny CA, Lahoti TS, Laurenzana EM, Omiecinski CJ, Perdew GH (2010) Kynurenic acid is a potent endogenous aryl hydrocarbon receptor ligand that synergistically induces interleukin-6 in the presence of inflammatory signaling. Toxicol Sci. 115:89-97. Engel J (2001) Mesial temporal lobe epilepsy: what have we learned? Neuroscientist. 7:340-352. Engel, J (2001) Mesial temporal lobe epilepsy: what have we learned? Neuroscientist. 7:340-352. Guillemin G, Kerr SJ, Smythe GA, Smith DG, Kapoor V, Armati PJ, Croitoru J, Brew BJ (2001) Kynurenine pathway meta- 
bolism in human astrocytes: a paradox for neuronal protection. J Neurochem. 78:842-853. Hilmas C, Pereira EF, Alkondon M, Rassoulpour A, Schwarcz R, Albuquerque EX (2001) The brain metabolite kynurenic acid inhibits alpha7 nicotinic receptor activity and increases non-alpha7 nicotinic receptor expression: physiopathological implications. J Neurosci. 21:7463-7473. Ito M, Seki T, Takuma, Y (2000) Current therapy of West syndrome in Japan. J Child. Neurol. 15:424-428. Jaeger B, Abeling NG, Salomons GS, Struys EA, SimasMendes M, Geukers VG, Poll-The BT (2016) Pyridoxine responsive epilepsy caused by a novel homozygous PNPO mutation. Mol Genet Metab Rep. 10:60-63. Jallon P, Loiseau P, Loiseau J (2001) Newly diagnosed unprovoked epileptic seizures: presentation at diagnosis in CAROLE study. Coordination Active du Réseau Observatoire Longitudinal de l' Epilepsie. Epilepsia. 42:464-475. Jin SH, Jeong W, Chung CK (2015) Mesial temporal lobe epilepsy with hippocampal sclerosis is a network disorder with altered cortical hubs. Epilepsia. 56:772-779. Josephson CB, Dykeman J, Fiest KM, Liu X, Sadler RM, Jette N, Wiebe S (2013) Systematic review and meta-analysis of standard vs selective temporal lobe epilepsy surgery. Neurology. 80:1669-1676. Kakkar A, Biswas A, Goyal N, Suri V, Sharma MC, Gupta D, Julka PK, Sarkar C (2016) The Expression of Cyclin D1, VEGF, EZH2, and H3K27me3 in atypical teratoid/rhabdoid tumors of the CNS: a possible role in targeted therapy. Appl Immunohistochem Mol Morphol. 24:729-737. Kamiński RM, Zielińska E, Dekundy A, van Luijtelaar G, Turski W (2003) Deficit of endogenous kynurenic acid in the frontal cortex of rats with a genetic form of absence epilepsy. Pol J Pharmacol. 55:741-746. Kessler M, Terramani T, Lynch G, Baudry M (1989) A glycine site associated with NMDA receptors: characterisation and identification of a new class of antagonist. J Neurochem. 52:1319-1328. Kuo MF, Wang HS (2002) Pyridoxal phosphate responsive epilepsy, with resistance to pyridoxine. Pediatr Neurol. 26:146-147. Kwan P, Arzimanoglou A, Berg AT, Brodie MJ, Hauser WA, Mathern G, Moshé SL, Perucca E, Wiebe S, French J (2010) Definition of drug resistant epilepsy: consensus proposal by the ad hoc Task Force of the ILAE Commission on Therapeutic Strategies. Epilepsia 51:1069-77. Livak KJ, Schmittgen TD (2001) Analysis of relative gene expression data using real-time quantitative PCR and the 2(-Delta Delta C(T)) Method. Methods. 25:402-408. Maciejak P. Szyndler J, Turzyńska D, Sobolewska A, Taracha E, Skórzewska A, Lehner M, Bidziński A, Płaźnik A (2008) Time course of changes in the concentration of kynurenic acid in the brain of pentylenetetrazol-kindled rats. Brain Res Bull. 78:299-305. Moroni F, Cozzi A, Sili M, Mannaioni G (2012) Kynurenic acid: a metabolite with multiple actions and multiple targets in brain and periphery. J Neural Transm. 119:133-139. Musayev FN, Di Salvo ML, Saavedra M, Contestabile R, Ghatke M, Haynes A, Schirch V, Safo MK (2009) Molecular basis of reduced pyridoxine 5'-phosphate oxidase catalytic activity in neonatal epileptic encephalopathy disorder. J Biol Chem. 284:30949-30956. Nürnberger S, Lindner C, Maier J, Strohmeier K, Wurzer C, Slezak P, Suessner S, Holnthoner W, Redl H, Wolbank S, Priglinger E (2019) Adipose-tissue-derived therapeutic cells in their natural environment as an autologous cell therapy strategy: the microtissue-stromal vascular fraction. Eur Cell Mater. 37:113-133 Parsons CG, Danysz W, Quack G, Hartmann S, Lorenz B, Wollenburg C, Baran L, Przegalinski E, Kostowski W, Krzascik P, Chizh B, Headley PM (1997) Novel systemically active antagonists of the glycine site of the N-methyl-D-aspartate receptor: electrophysiological, biochemical and behavioral characterization. J Pharmacol Exp Ther. 283:1264-1275 Perkins M, Stone T (1984) Actions of excitatory amino acids and kynurenic acid in the primate hippocampus: A preliminary study. Neurosci Lett. 52:335-340. Pocivavsek A, Wu HQ, Potter MC, Elmer GI, Pellicciari R, Schwarcz R (2011) Fluctuations in endogenous kynurenic acid control hippocampal glutamate and memory. Neuropsychopharmacology. 36:2357-2367. Potter M, Elmer G, Bergeron R, Albuquerque E, Guidetti P, Wu H, Schwarcz R (2010) Reduction of endogenous kynurenic acid formation enhances extracellular glutamate, hippocampal plasticity, and cognitive behavior. Neuropsychopharmacology. 35:1734-1742. Rassoulpour A, Wu HQ, Ferré S, Schwarcz R (2005) Nanomolar concentrations of kynurenic acid reduce extracellular dopamine levels in the striatum. J Neurochem. 93:762-765. Roy S, Sharma HP, Nag TC, Velpandian T, Upadhyay AD, Mathur R, Jain S (2014) BDNF mediated activity dependent maturation of visual Wulst following prenatal repetitive auditory stimulation at a critical developmental period in domestic chicks (Gallus domesticus). Brain Res Bull. 109:99108. Scharfman HE (2007) The neurobiology of epilepsy. Curr Neurol Neurosci. Rep. 7:348-354. Schwarcz R, Bruno J, Muchowski P, Wu H (2012) Kynurenines in the mammalian brain: when physiology meets pathology. Nat Rev Neurosci. 13:465-477. Szyndler J, Maciejak P, Turzyńska D, Sobolewska A, Walkowiak J, Płaźnik A (2012) The effects of electrical hippocampal kindling of seizures on amino acids and kynurenic 
acid concentrations in brain structures. J Neural Transm. 119:141-149. Tripathi M, Ray S, Chandra PS (2016) Presurgical evaluation for drug refractory epilepsy. Int J Surg. 36:405-410. Turski WA, Gramsbergen JB, Traitler H, Schwarcz R (1989) Rat Brain Slices Produce and Liberate Kynurenic Acid upon Exposure to L-Kynurenine. J Neurochem. 52:1629-1636. Watanabe K (1995) Medical treatment of West syndrome in Japan. J Child Neurol. 10:143-147. Wu H, Turski W, Ungerstedt U, Sahwarcz R (1991) Systemic Kainic Acid Administration in Rats: Effects on Kynurenic Acid Production in Vitro and in Vivo. Exp Neurol. 113:47-52. Xiao LD, Luo XB, Pi LG, Tang AG (2008) Simultaneous determination of kynurenine and kynurenic acid concentrations in human serum by HPLC with dual wavelengths fluorescence detection. Clin Chim Acta. 395:178-180 Yamamoto H, Murakami H, Horiguchi K, Egawa B (1995) Studies on cerebrospinal fluid kynurenic acid concentrations in epileptic children. Brain Dev. 17:327-329. Yamamoto H, Shindo I, Egawa B, Horiguchi K (1994) Kynurenic acid is decreased in cerebrospinal fluid of patients with infantile spasms. Pediatr Neurol. 10:9-12.

Table 1. Characteristics of PSCs (frequency, amplitude, rise time, decay time constant) recorded from pyramidal neurons in non-seizure controls and hippocampal samples obtained from patients with MTLEHS.

\begin{tabular}{|c|c|}
\hline Parameters & Non-seizure Control $(n=$ \\
\hline $\begin{array}{l}\text { Spontaneous EPSCs } \\
\text { EPS }\end{array}$ & Spontaneous EPSCs \\
\hline Frequency $(\mathrm{Hz})$ & $0.68 \pm 0.01$ \\
\hline Amplitude (pA) & $12.05 \pm 0.16$ \\
\hline Rise time (ms) & $1.9 \pm 0.7$ \\
\hline Decay time constant $\left(\tau_{d}, \mathrm{~ms}\right)$ & $9.6 \pm 1.4$ \\
\hline Spontaneous IPSCs & Spontaneous IPSCs \\
\hline Frequency $(\mathrm{Hz})$ & $1.76 \pm 0.04$ \\
\hline Amplitude (pA) & $22.86 \pm 0.41$ \\
\hline Rise time (ms) & $2.9 \pm 0.7$ \\
\hline Decay time constant $\left(\tau_{d}, \mathrm{~ms}\right)$ & $32.4 \pm 3.8$ \\
\hline 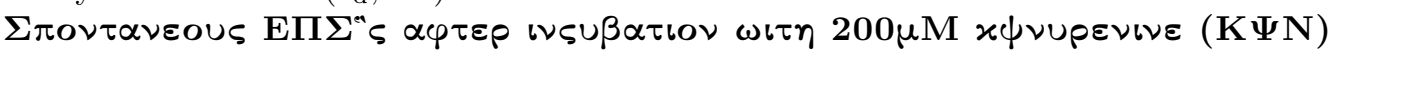 & $\begin{array}{l}\Sigma \pi o \nu \tau \alpha \nu \varepsilon o \cup \varsigma \text { EП } \Sigma^{\prime \prime} \varsigma \alpha \\
\text { Non-seizure Control }(n=\end{array}$ \\
\hline Frequency $(\mathrm{Hz})$ & $0.67 \pm 0.02$ \\
\hline Amplitude (pA) & $12.53 \pm 0.23$ \\
\hline Rise time (ms) & $2.0 \pm 0.3$ \\
\hline \multirow[t]{2}{*}{ Decay time constant $\left(\tau_{d}, \mathrm{~ms}\right)$} & $9.3 \pm 1.2$ \\
\hline & MTLE-HS $(n=16)$ \\
\hline Frequency $(\mathrm{Hz})$ & $1.00 \pm 0.07$ \\
\hline Amplitude (pA) & $15.39 \pm 0.20$ \\
\hline Rise time (ms) & $2.2 \pm 0.3$ \\
\hline Decay time constant $\left(\tau_{\mathrm{d}}, \mathrm{ms}\right)$ & $9.0 \pm 1.1$ \\
\hline 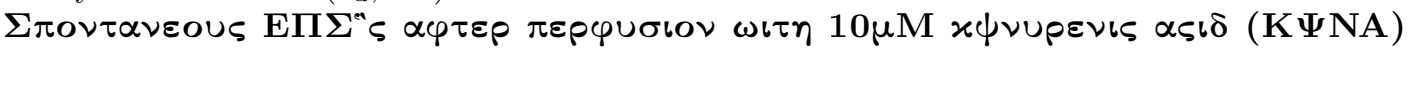 & $\begin{array}{l}\Sigma \pi o \nu \tau \alpha \nu \varepsilon o \cup \varsigma \text { Eח } \Sigma^{\prime \prime} \varsigma \alpha \\
\text { Non-seizure Control }(n=\end{array}$ \\
\hline Frequency $(\mathrm{Hz})$ & $0.65 \pm 0.05$ \\
\hline Amplitude (pA) & $12.51 \pm 0.26$ \\
\hline Rise time (ms) & $1.9 \pm 0.7$ \\
\hline \multirow[t]{2}{*}{ Decay time constant $\left(\tau_{\mathrm{d}}, \mathrm{ms}\right)$} & $9.7 \pm 1.4$ \\
\hline & MTLE-HS $(n=10)$ \\
\hline Frequency $(\mathrm{Hz})$ & $1.06 \pm 0.09$ \\
\hline Amplitude (pA) & $16.29 \pm 0.30$ \\
\hline Rise time (ms) & $2.0 \pm 0.6$ \\
\hline Decay time constant $\left(\tau_{d}, \mathrm{~ms}\right)$ & $9.6 \pm 0.8$ \\
\hline
\end{tabular}


Data are presented as a mean \pm SEM. ${ }^{* *} \mathrm{P}<0.01,{ }^{* * *} \mathrm{P}<0.001$. Two tailed unpaired T-test/ Mann Whitney Test

\section{Figure Legends}

Figure 1: Histology and immunohistochemistry reveal features of hippocampal sclerosis. Photomicrographs showing histology of the MTLE-HS hippocampal samples and non-seizure control tissue sections. Hematoxylin \& Eosin stained MTLE-HS hippocampal specimens show (a) loss of neurons, (b) highlighted by NeuN, (c) GFAP shows reactive astrogliosis (arrow heads). Autopsy control specimens show (d) no loss of neurons in H \& E stained sections, (e) supported by NeuN IHC, (f) no reactive gliosis as revealed in GFAP IHC.

Figure 2: Quantitative estimation of tryptophan-kynurenine pathway metabolites. (a) concentration of tryptophan (autopsy control, $\mathrm{n}=6$, MTLE-HS, $\mathrm{n}=20 ; 1.068 \pm 0.28 \mathrm{ng} / \mathrm{mg}$ of wet tissue in autopsy control vs $0.086 \pm 0.013 \mathrm{ng} / \mathrm{mg}$ of wet tissue in MTLE-HS), (b) concentration of kynurenine (autopsy control, $\mathrm{n}=10$, MTLE-HS, $\mathrm{n}=43 ; 2.895 \pm 1.05 \mathrm{ng} / \mathrm{mg}$ of wet tissue in autopsy control vs $1.74 \pm 0.57 \mathrm{ng} / \mathrm{mg}$ of wet tissue in MTLE-HS), (c) tryptophan kynurenine ratio (autopsy control, $\mathrm{n}=6$, MTLE-HS, $\mathrm{n}=20 ; 1.58 \pm$ 0.80 in autopsy control vs $0.05 \pm 0.009$ in MTLE-HS), (d) concentration of kynurenic acid (autopsy control, $\mathrm{n}=10$, MTLE-HS, $\mathrm{n}=43 ; 0.184 \pm 0.046 \mathrm{ng} / \mathrm{mg}$ of wet tissue in autopsy control vs $0.0289 \pm 0.0039 \mathrm{ng} / \mathrm{mg}$ of wet tissue in MTLE-HS), (e) concentration of quinolinic acid (autopsy control, $n=10$, MTLE-HS, $n=14$; $11.67 \pm 2.98 \mathrm{ng} / \mathrm{mg}$ of wet tissue in autopsy control vs $39.22 \pm 9.23 \mathrm{ng} / \mathrm{mg}$ of wet tissue in MTLE-HS), (f) quinolinic acid kynurenic acid ratio (autopsy control, $n=10$, MTLE-HS, $n=14 ; 90.34 \pm 28.97$ in autopsy control vs $242.0 \pm 20.25$ in MTLE-HS), (g) concentration of glutamate (autopsy control, $\mathrm{n}=10$, MTLE-HS, $\mathrm{n}=12 ; 12.08 \pm 3.01 \mathrm{ng} / \mu \mathrm{g}$ protein in autopsy control vs $10.57 \pm 4.7 \mathrm{ng} / \mu \mathrm{g}$ protein in MTLE-HS), (h) concentration of GABA (control, $\mathrm{n}=10$, MTLE-HS, $\mathrm{n}=12 ; 1.18 \pm 0.32 \mathrm{ng} / \mu \mathrm{g}$ protein in autopsy control vs 1.15 $\pm 0.16 \mathrm{ng} / \mu \mathrm{g}$ protein in MTLE-HS), (i) glutamate GABA ratio (autopsy control, $\mathrm{n}=10$, MTLE-HS, $\mathrm{n}=12$; $15.34 \pm 4.98$ in autopsy control vs $8.46 \pm 2.02$ in MTLE-HS) in autopsy control samples and hippocampal samples obtained patients with MTLE-HS. (j) Concentration ofde novo synthesis of kynurenic acid (nonseizure control, $\mathrm{n}=18$, MTLE-HS, $\mathrm{n}=26 ; 5.28 \pm 1.13 \mathrm{ng} / \mathrm{mg}$ of wet tissue in non-seizure control vs $0.10 \pm 0.03$ $\mathrm{ng} / \mathrm{mg}$ of wet tissue in MTLE-HS) and (k) concentration ofde novo synthesis of quinolinic acid (non-seizure control, $\mathrm{n}=12$, MTLE- MTLE-HS, $\mathrm{n}=12 ; 8.66 \pm 2.28 \mathrm{ng} / \mathrm{mg}$ of wet tissue in non-seizure control vs $22.33 \pm$ $7.47 \mathrm{ng} / \mathrm{mg}$ of wet tissue in MTLE-HS) in non-seizure control samples and hippocampal samples obtained patients with MTLE-HS. Data are presented as a mean \pm SEM. ${ }^{*}$ denotes $\mathrm{p}<0.05,{ }^{* *} \mathrm{p}<0.01,{ }^{* * *} \mathrm{p}<0.001$. Mann Whitney Test.

Figure 3: Spontaneous excitatory postsynaptic currents were enhanced in MTLE-HS hippocampal samples but spontaneous inhibitory postsynaptic currents were unaffected. (a) Sample recordings of spontaneous EPSCs recorded from pyramidal neurons in the cortical sample obtained from non-seizure controls. Inset shows a single EPSC event at an expanded time scale. The second trace shows absence of any spontaneous EPSCs following perfusion of the slice with glutamate receptor antagonists APV $(50 \mu \mathrm{M})$ and CNQX $(10 \mu \mathrm{M})$ for 10-min proving that these events are glutamate receptor. The bottom trace shows sample recordings of spontaneous EPSCs recorded from pyramidal neurons in the hippocampal sample obtained from patients with MTLE-HS. Plots represent data from eight neurons from eight patients for non-seizure control, nine neurons from the hippocampal samples of nine MTLE-HS patients. (b) In the hippocampal samples, cumulative distribution of inter-event interval displaced toward lower intervals and (c) that of peak amplitude displaced towards longer amplitude. (d) Sample recordings of spontaneous IPSCs recorded from pyramidal neurons in the cortical sample obtained from non-seizure controls and the hippocampal samples obtained from patients with MTLE-HS. The bottom trace shows absence of any spontaneous IPSCs following perfusion of the slice with $\mathrm{GABA}_{\mathrm{A}}$ receptor antagonist bicuculline $(10 \mu \mathrm{M})$ for 10 -min proving that these events are $\mathrm{GABA}_{\mathrm{A}}$ receptor mediated. Plots represent data from five neurons from five patients for non-seizure control, five neurons from the hippocampal samples of five MTLE-HS patients. (e) Cumulative distribution of inter-event interval and (f) peak amplitude of the IPSCs also did not alter. (g) Frequency and amplitude ratio of EPSC/IPSC both were significantly higher in the hippocampal samples. 
Data represented as mean \pm SEM. ${ }^{*} \mathrm{p}<0.01,{ }^{* *} \mathrm{p}<0.01,{ }^{* * *} \mathrm{p}<0.001$. Two tailed unpaired T-test/ Mann Whitney Test.

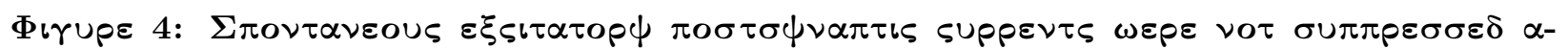

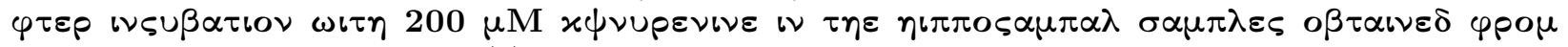
$\pi \alpha \tau \iota \varepsilon \nu \tau \varsigma \omega \iota \tau \eta ~ M T \Lambda E-H \Sigma$. (a) Sample recordings of spontaneous EPSCs recorded from pyramidal neurons in cortical sample obtained from non-seizure controls before and after incubation with200 $\mu \mathrm{M}$ kynurenine for 85 mins. Plots represent data from six neurons from six patients for non-seizure control. (b) Cumulative distribution of inter-event interval displaced towards longer intervals while that of (c) peak amplitude displaced towards shorter amplitude. (d) Sample recordings of spontaneous EPSCs recorded from pyramidal neurons in hippocampus sample obtained from MTLE-HS patients without and with $200 \mu \mathrm{M}$ kynurenine. Plots represent data from sixteen neurons from sixteen patients for MTLE-HS. (e) Cumulative distribution of inter-event interval and (f) peak amplitude did not displace. (g) Percentage reduction of frequency was significantly reduced in the hippocampal samples after incubation with $200 \mu \mathrm{M}$ kynurenine. Data represented as mean \pm SEM. ${ }^{* * *} \mathrm{p}<0.001$. Two tailed unpaired T-test/ Mann Whitney Test.

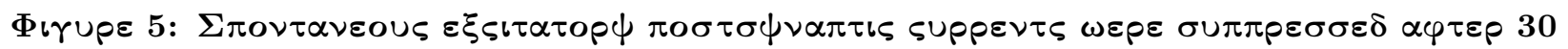

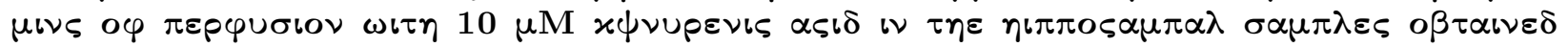

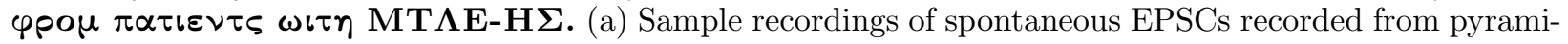
dal neurons in cortical sample obtained from non-seizure controls without and with $10 \mu \mathrm{M}$ kynurenic acid. Plots represent data from six neurons from six patients for non-seizure control. (b) Cumulative distribution of inter-event interval displaced towards longer intervals while that of (c) peak amplitude displaced towards shorter amplitude. (d) Sample recordings of spontaneous EPSCs recorded from pyramidal neurons in the hippocampus sample obtained from patients with MTLE-HS without and with $10 \mu \mathrm{M}$ kynurenic acid. Plots represent data from ten neurons from ten patients for MTLE-HS. (e) Cumulative distribution of inter-event interval displaced towards longer intervals while that of (f) peak amplitude displaced towards shorter amplitude. (g) Percentage reduction of frequency was significantly increased in the hippocampal samples after perfusion with $10 \mu \mathrm{M}$ kynurenic acid. (h) Perfusion with $50 \mu \mathrm{M}$ APV significantly reduced frequency of spontaneous excitatory postsynaptic currents in both non-seizure controls and hippocampal samples ( $\mathrm{n}=6$; $0.98 \pm 0.018 \mathrm{~Hz}$ without APV vs $0.41 \pm 0.25 \mathrm{~Hz}$ with APV). (i) Percentage reduction of frequency was significantly increased in the hippocampal samples after perfusion with $50 \mu \mathrm{M}$ APV respectively. (j) Within the hippocampal samples, percentage reduction of frequency was significantly increased after kynurenic acid treatment in comparison to kynurenine incubation and after APV treatment in comparison to kynurenic acid. Data represented as mean \pm SEM. ${ }^{* * *} \mathrm{p}<0.001, \# \mathrm{p}<0.05$. Two tailed unpaired T-test/ Mann Whitney Test/one way ANOVA with Dunn's posthoc test.

Figure 6: Altered enzyme expression levels of tryptophan-kynurenine pathway contributes to reduced KYNA levels in the hippocampal samples obtained from patients with MTLE-HS. (a) KAT II mRNA expression did not alter significantly (autopsy control, $\mathrm{n}=6$, MTLE-HS, $\mathrm{n}=12 ; \Delta \mathrm{C}_{\mathrm{T}}$ $1.49 \pm 0.25$ in autopsy control vs $\Delta \mathrm{C}_{\mathrm{T}} 1.43 \pm 0.44$ in MTLE-HS) but (b) protein level expression was significantly decreased in the hippocampal samples (autopsy control, $\mathrm{n}=8$, MTLE-HS, $\mathrm{n}=10$; normalised expression $0.90 \pm 0.03$ in autopsy control vs normalised expression $0.76 \pm 0.062$ in MTLE-HS). (c) IDO mRNA expression (autopsy control, $\mathrm{n}=6$, MTLE-HS, $\mathrm{n}=10 ; \Delta \mathrm{C}_{\mathrm{T}} 11.12 \pm 1.0$ in autopsy control vs $\Delta \mathrm{C}_{\mathrm{T}}$ $1.67 \pm 0.89$ in MTLE-HS) as well as (d) protein expression (autopsy control, $\mathrm{n}=8$, MTLE-HS, $\mathrm{n}=10 ; 246.4$ $\pm 11.36 \mathrm{ng} / \mathrm{ml}$ in autopsy control vs $294.7 \pm 2.77 \mathrm{ng} / \mathrm{ml}$ in MTLE-HS) both significantly increased in the hippocampal samples. (e) KMO mRNA (autopsy control, $\mathrm{n}=6$, MTLE-HS, $\mathrm{n}=12 ; \Delta \mathrm{C}_{\mathrm{T}} 7.11 \pm 0.81$ in autopsy control vs $\Delta \mathrm{C}_{\mathrm{T}} 5.95 \pm 0.49$ in MTLE-HS) as well as (f) protein level expression (autopsy control, $\mathrm{n}=8$, MTLE-HS, $\mathrm{n}=10$; normalised expression $0.79 \pm 0.073$ in autopsy control vs normalised expression 0.74 \pm 0.049 in MTLE-HS) did not alter. (g) PLP concentration was significantly reduced in the hippocampal samples (autopsy control, $\mathrm{n}=6$, MTLE-HS, $\mathrm{n}=27 ; 5.03 \pm 1.01 \mathrm{ng} / \mathrm{mg}$ of wet tissue in autopsy control vs 2.04 $\pm 0.75 \mathrm{ng} / \mathrm{mg}$ of wet tissue in MTLE-HS). (h) mRNA expression of PNPO was significantly reduced in the hippocampal samples (autopsy control, $\mathrm{n}=6$, MTLE-HS, $\mathrm{n}=9 ; \Delta \mathrm{C}_{\mathrm{T}} 3.41 \pm 0.43$ in autopsy control vs $\Delta \mathrm{C}_{\mathrm{T}}$ $5.8 \pm 0.29$ in MTLE-HS). (i) Protein expression of PNPO was also significantly reduced in the hippocampal 
samples (autopsy control, $\mathrm{n}=8$, MTLE-HS, $\mathrm{n}=10$; normalised expression $0.58 \pm 0.042$ in autopsy control vs normalised expression $0.39 \pm 0.045$ in MTLE-HS). The Western blot images of KAT II, KMO, PNPO, and GAPDH represent single band of KAT II at the predicted size of $50 \mathrm{KDa}$, KMO at $45 \mathrm{KDa}$, PNPO at 30 $\mathrm{KDa}$ and GAPDH at $37 \mathrm{KDa}$. Data represented as mean \pm SEM. ${ }^{*} \mathrm{p}<0.05,{ }^{* *} \mathrm{p}<0.01,{ }^{* * *} \mathrm{p}<0.001$. Mann Whitney Test.

Figure 7: The concentration of metabolites in the hippocampal samples was correlated with seizure duration of patients with MTLE-HS.(a) Scatterplot shows inverse correlation (sample size 40; Spearman's non-parametric correlation, $\mathrm{r}=-0.4712$, $\mathrm{p}=0.0021$; line of best fit $\mathrm{Y}=-0.001385^{*} \mathrm{X}+0.06080$, slope $\left.=-0.001385 \pm 0.0004206, \mathrm{R}^{2}=0.2220, \mathrm{~F}=10.84, \mathrm{p}=0.0021\right)$ between concentration of KYNA in the hippocampal samples obtained from patients with MTLE-HS and seizure duration (years). (b) Scatterplot shows inverse correlation (sample size 26; Spearman's non-parametric correlation, $\mathrm{r}=-0.6173, \mathrm{p}=0.0008$; line of best fit $\mathrm{Y}=-0.009452^{*} \mathrm{X}+0.2413$, slope $=-0.009452 \pm 0.003495, \mathrm{R}^{2}=0.2336, \mathrm{~F}=7.313, \mathrm{p}=0.0124$ ) between de novo synthesis of KYNA in the hippocampal samples obtained from patients with MTLE-HS and seizure duration (years). (c) Scatterplot shows correlation (sample size 14; Spearman's non-parametric correlation, $\mathrm{r}=0.5423, \mathrm{p}=0.0476$; line of best fit $\mathrm{Y}=3.683^{*} \mathrm{X}-10.84$, slope $=3.683 \pm 1.135, \mathrm{R}^{2}=0.4675, \mathrm{~F}=10.53$, $\mathrm{p}=0.0070$ ) between concentration of QUIN in the hippocampal samples obtained from patients with MTLEHS and seizure duration (years). (d) Scatterplot shows no significant correlation (sample size 12; Spearman's non-parametric correlation, $\mathrm{r}=0.5750, \mathrm{p}=0.0542$ ) between de novosynthesis of QUIN in the hippocampal samples obtained from patients with MTLE-HS and seizure duration (years). (e) Scatterplot shows inverse correlation (sample size 29; Spearman's non-parametric correlation, $\mathrm{r}=-0.4647, \mathrm{p}=0.0111$; line of best fit $\mathrm{Y}=-0.04474 * \mathrm{X}+1.880$, slope $\left.=-0.04474 \pm 0.01921, \mathrm{R}^{2}=0.1673, \mathrm{~F}=5.425, \mathrm{p}=0.0276\right)$ between concentration of PLP in the hippocampal samples obtained from patients with MTLE-HS and seizure duration (years).
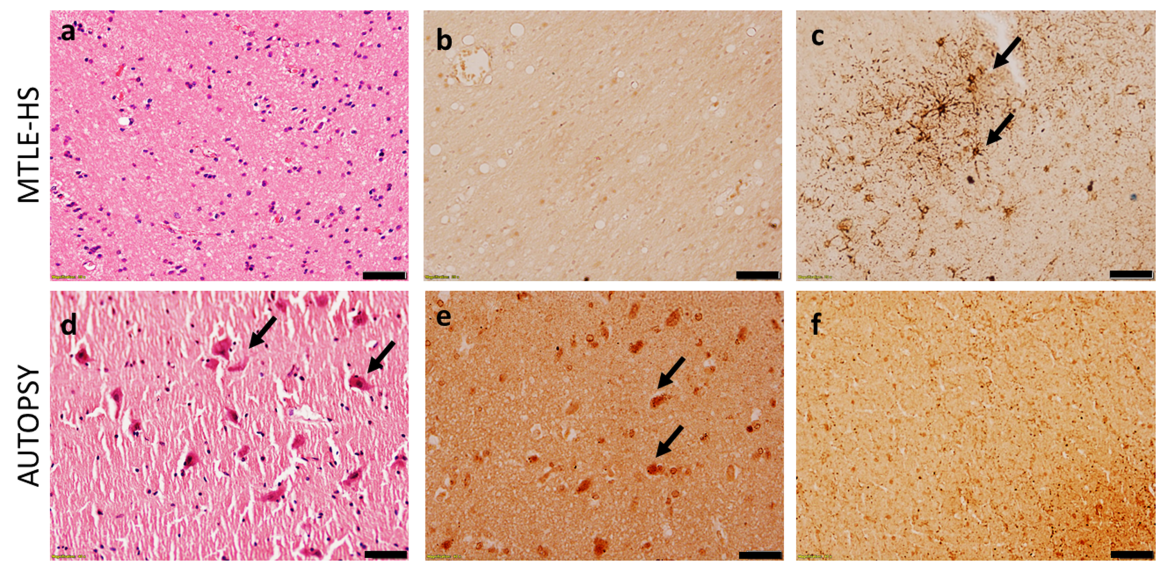

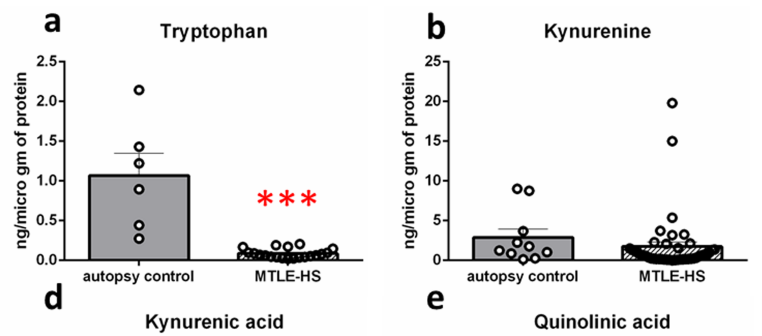

C Tryptophan Kynurenine ratio
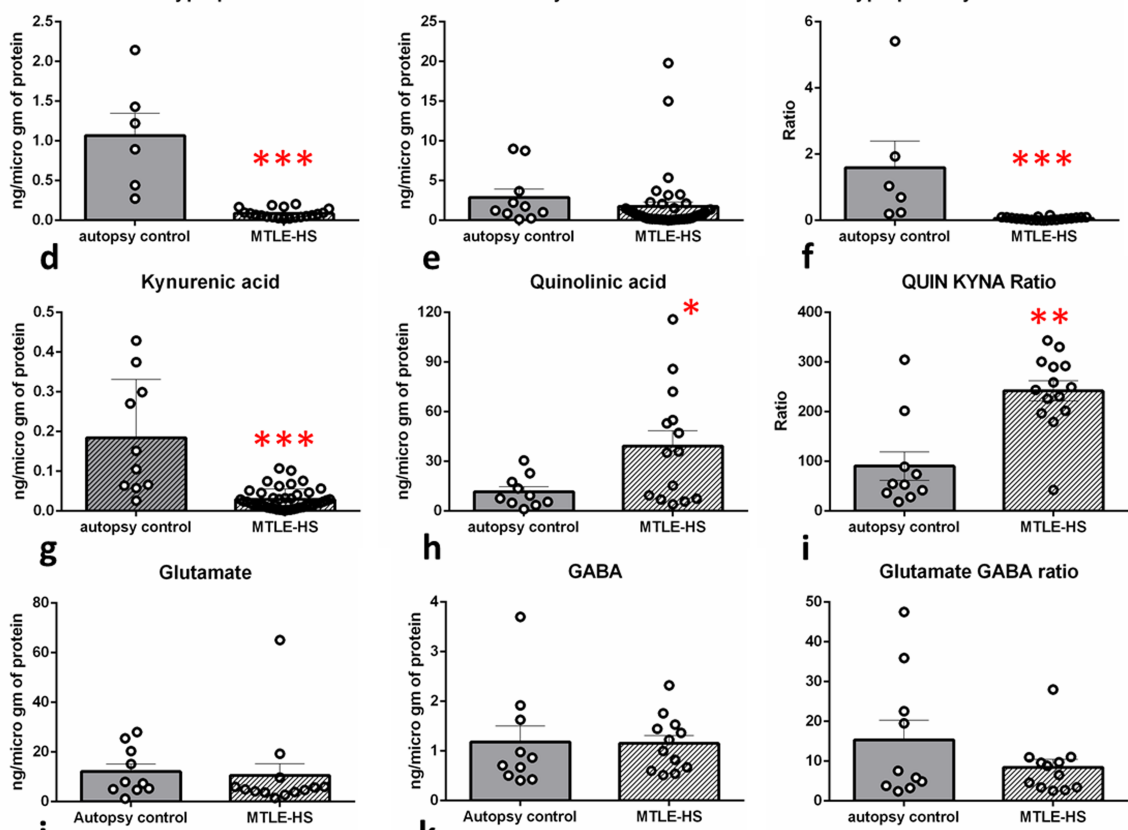

$$
\text { j }
$$

MTLE-HS
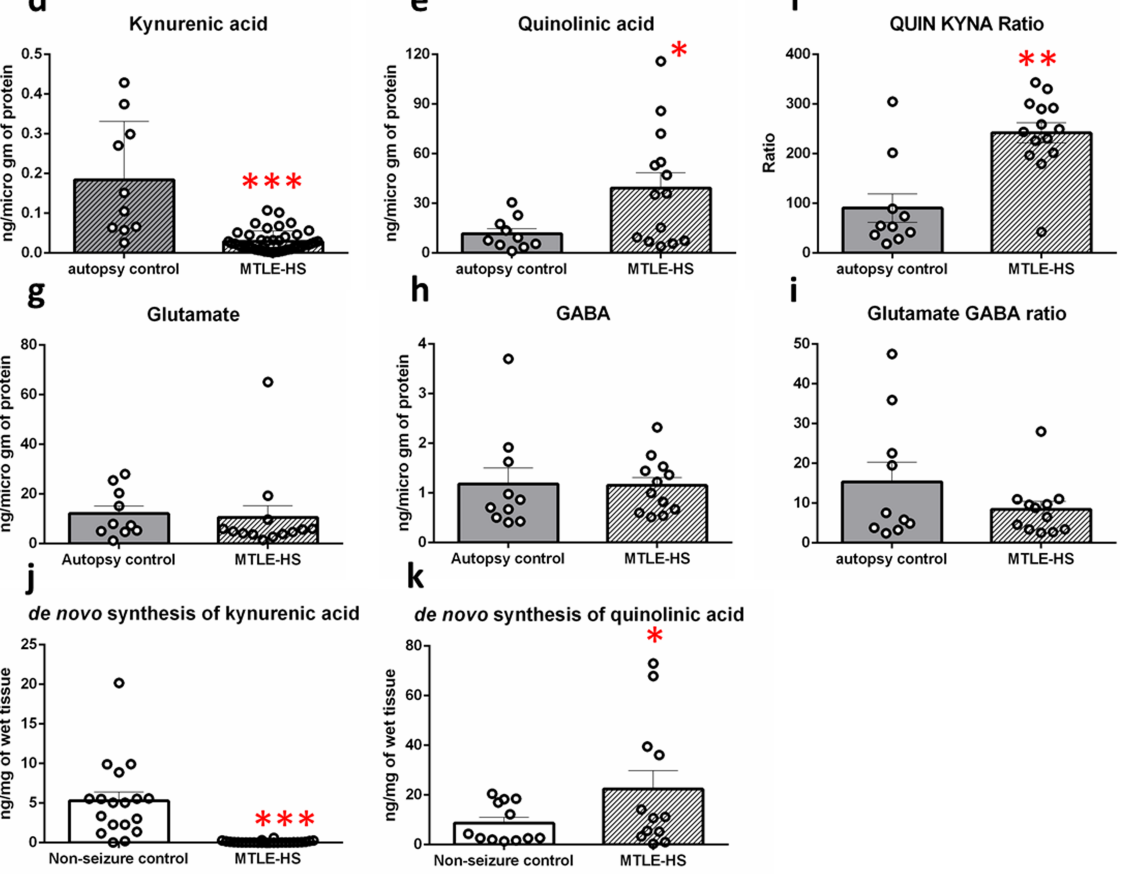
a

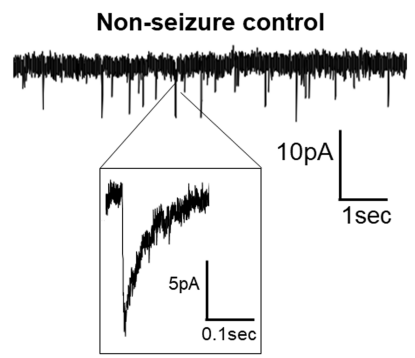

$50 \mu M A P V+10 \mu M C N Q X$

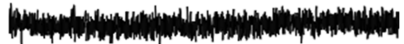

MTLE-HS hippocampus

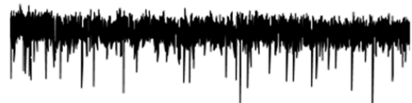

b

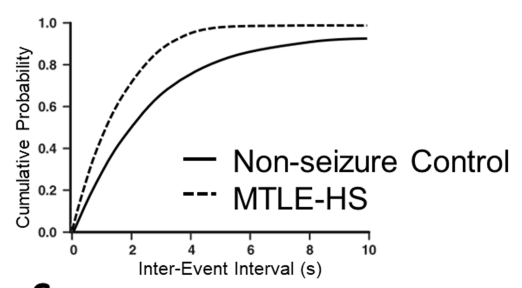

C

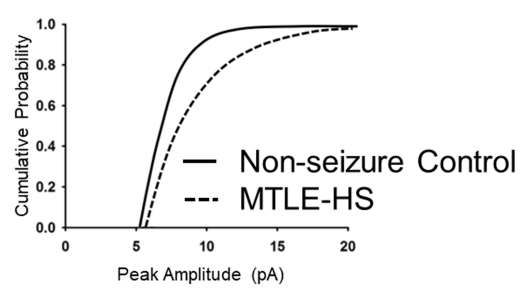

g

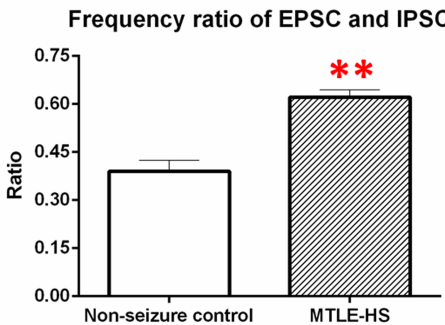

d

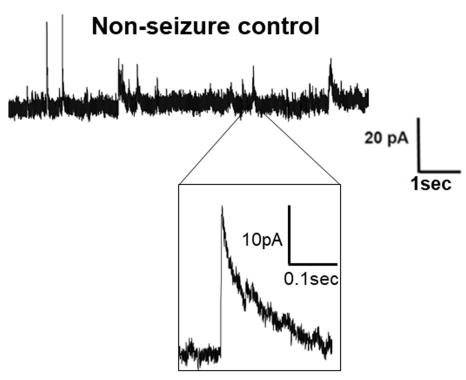

$10 \mu \mathrm{M}$ Bicuculline

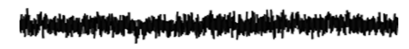

MTLE-HS hippocampus

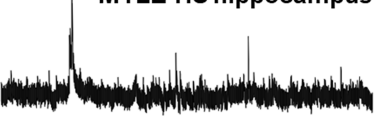

e

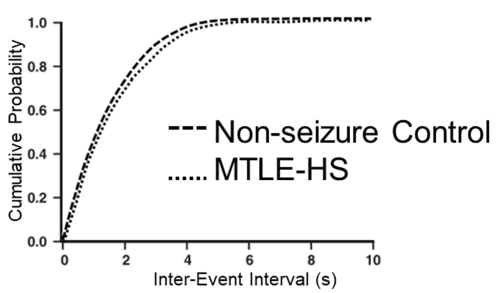

f

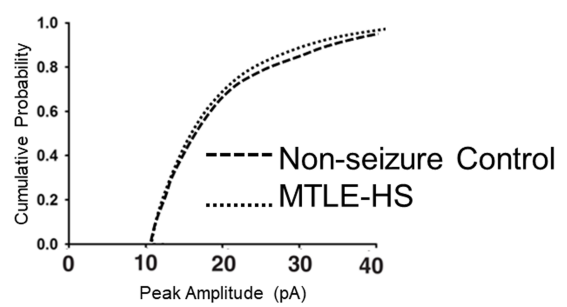

Amplitude ratio of EPSC and IPSC

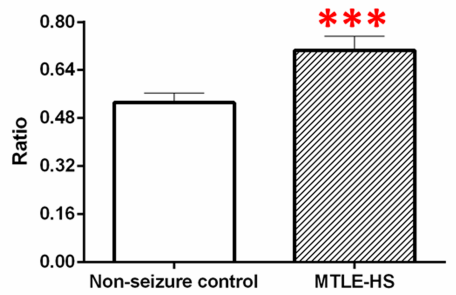



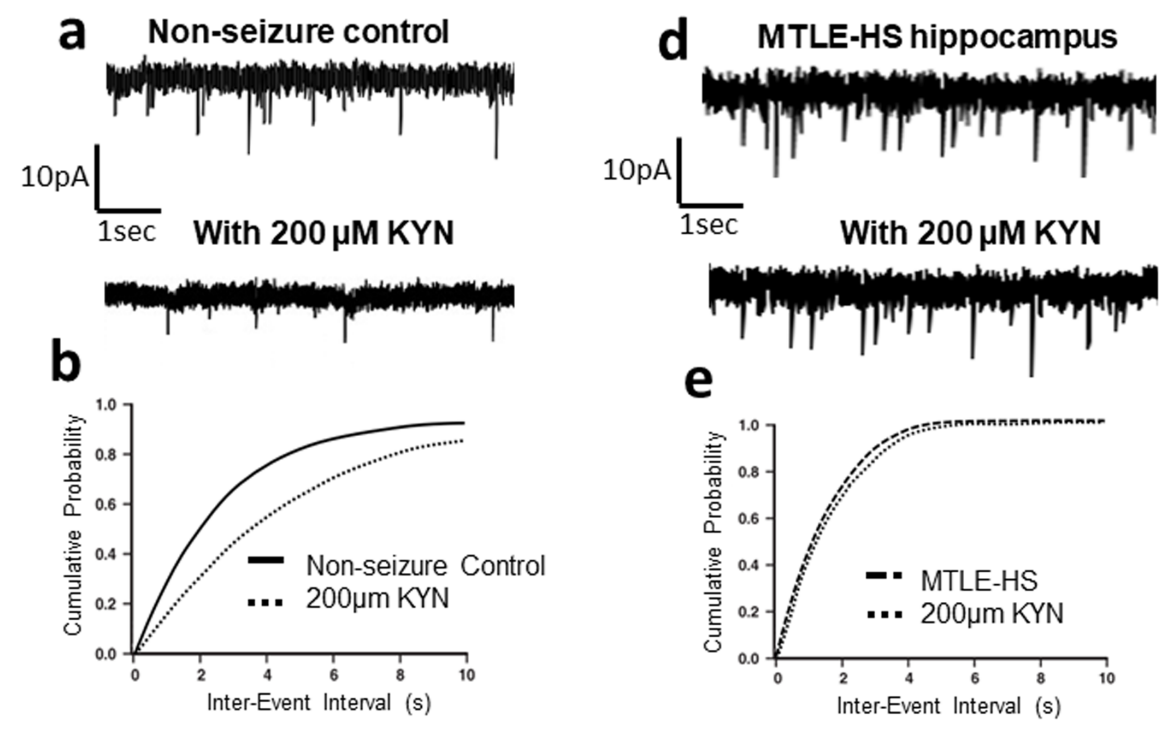

C
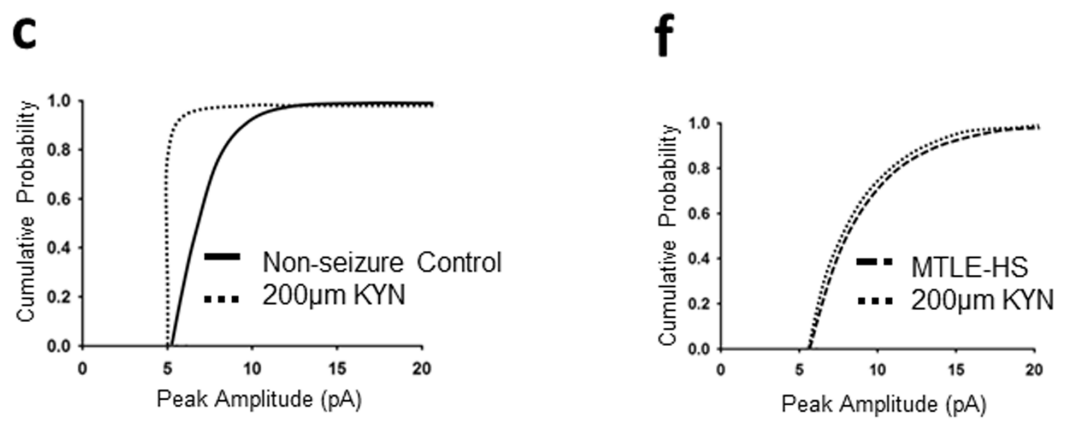

g Percentage frequency reduction with KYN

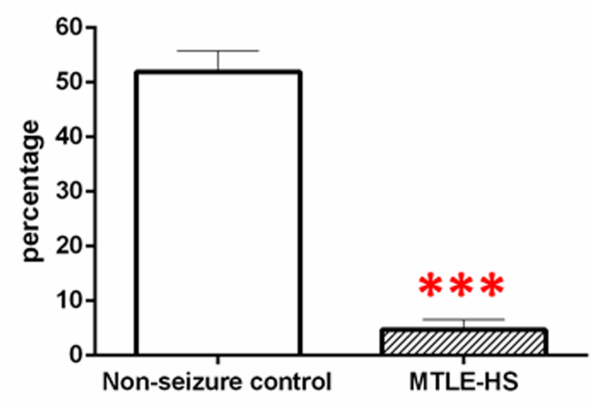



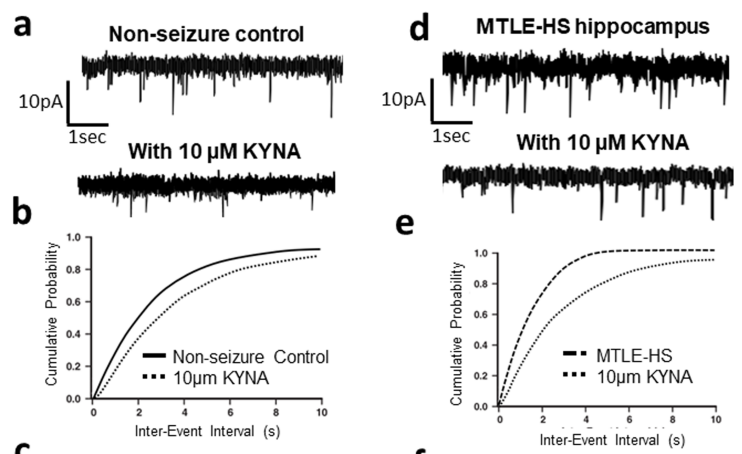

e
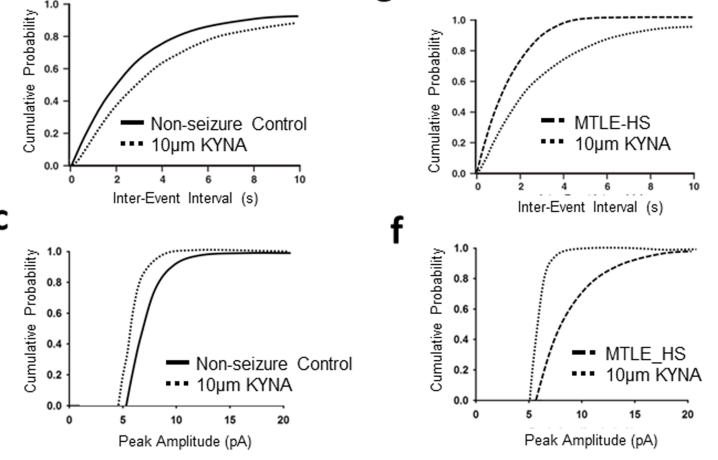

f

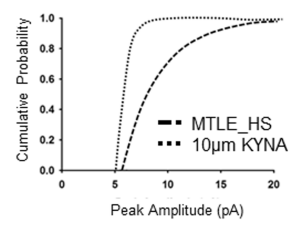

g Percentage frequency reduction with KYNA

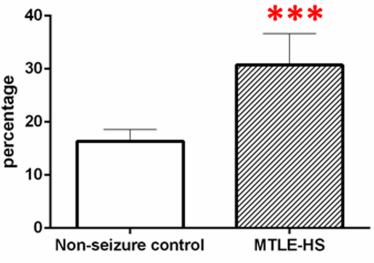

h

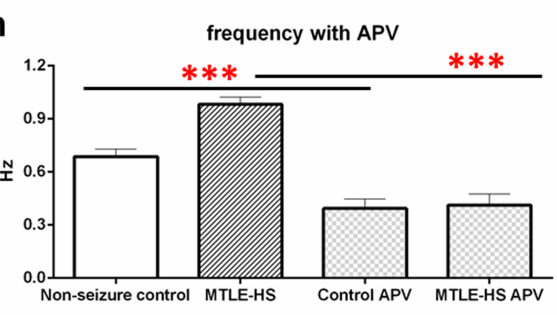

i Percentage frequency reduction with APV

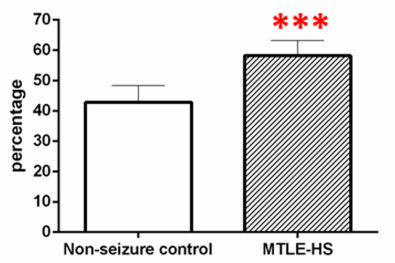

J

comparison of frequency reduction in MTLE-HS

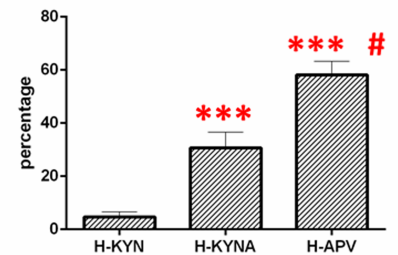



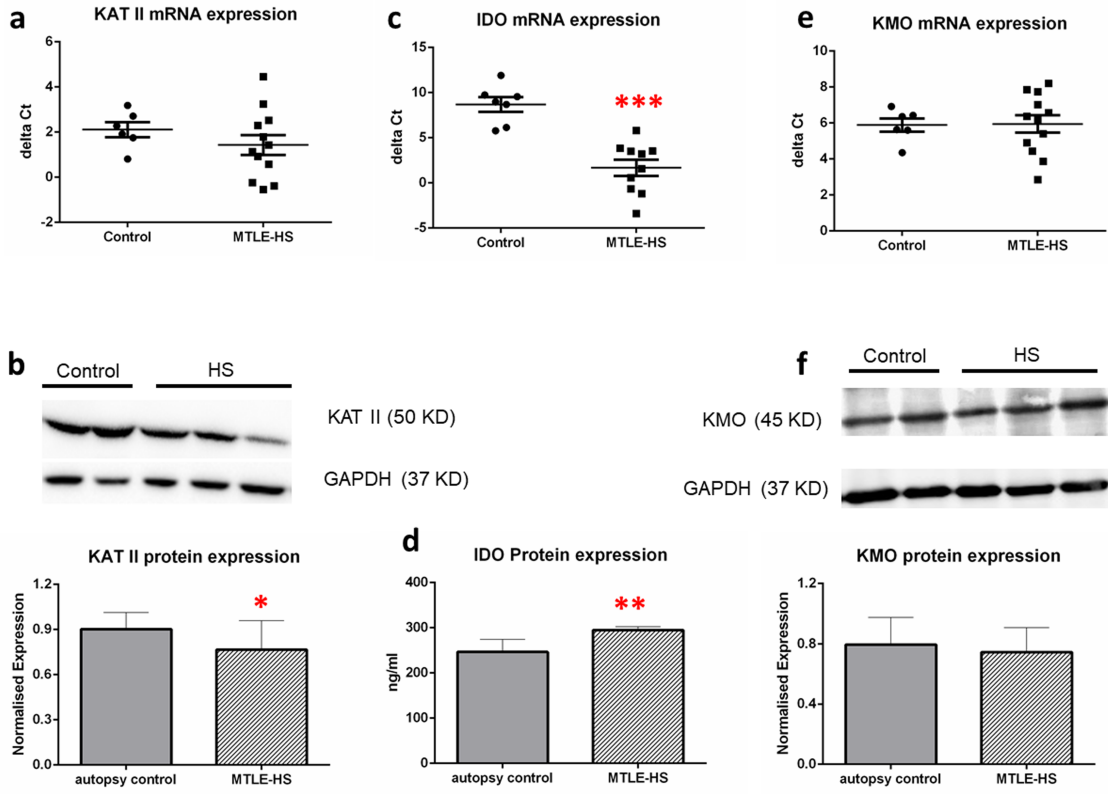

$$
\begin{aligned}
& \text { KAT ॥ (50 KD) } \\
& \text { GAPDH (37 KD) }
\end{aligned}
$$
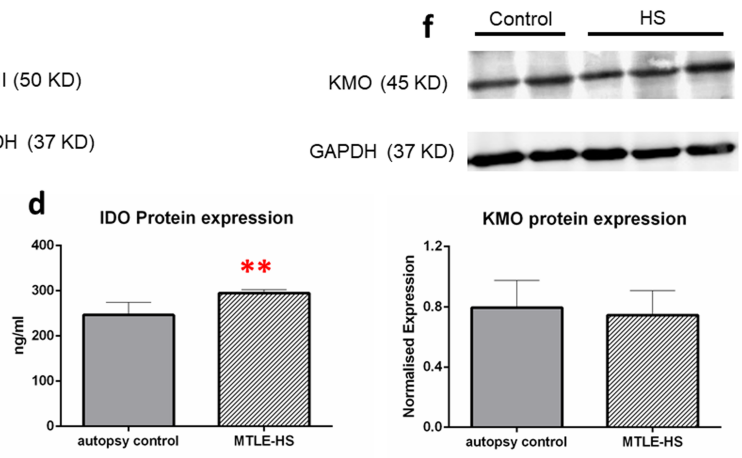

g

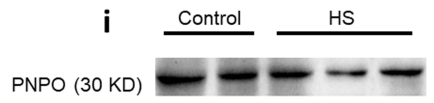

Pyridoxal Phosphate

GAPDH (37 KD)
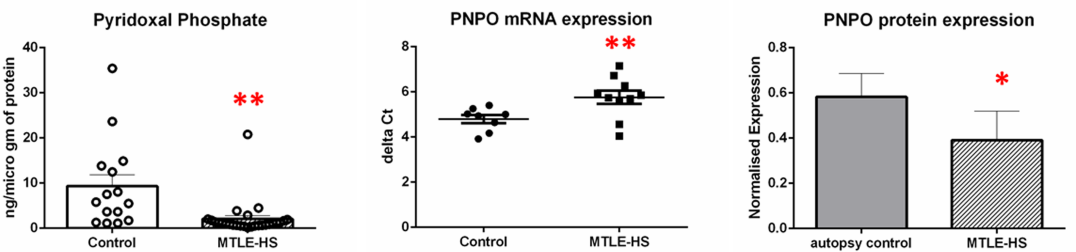

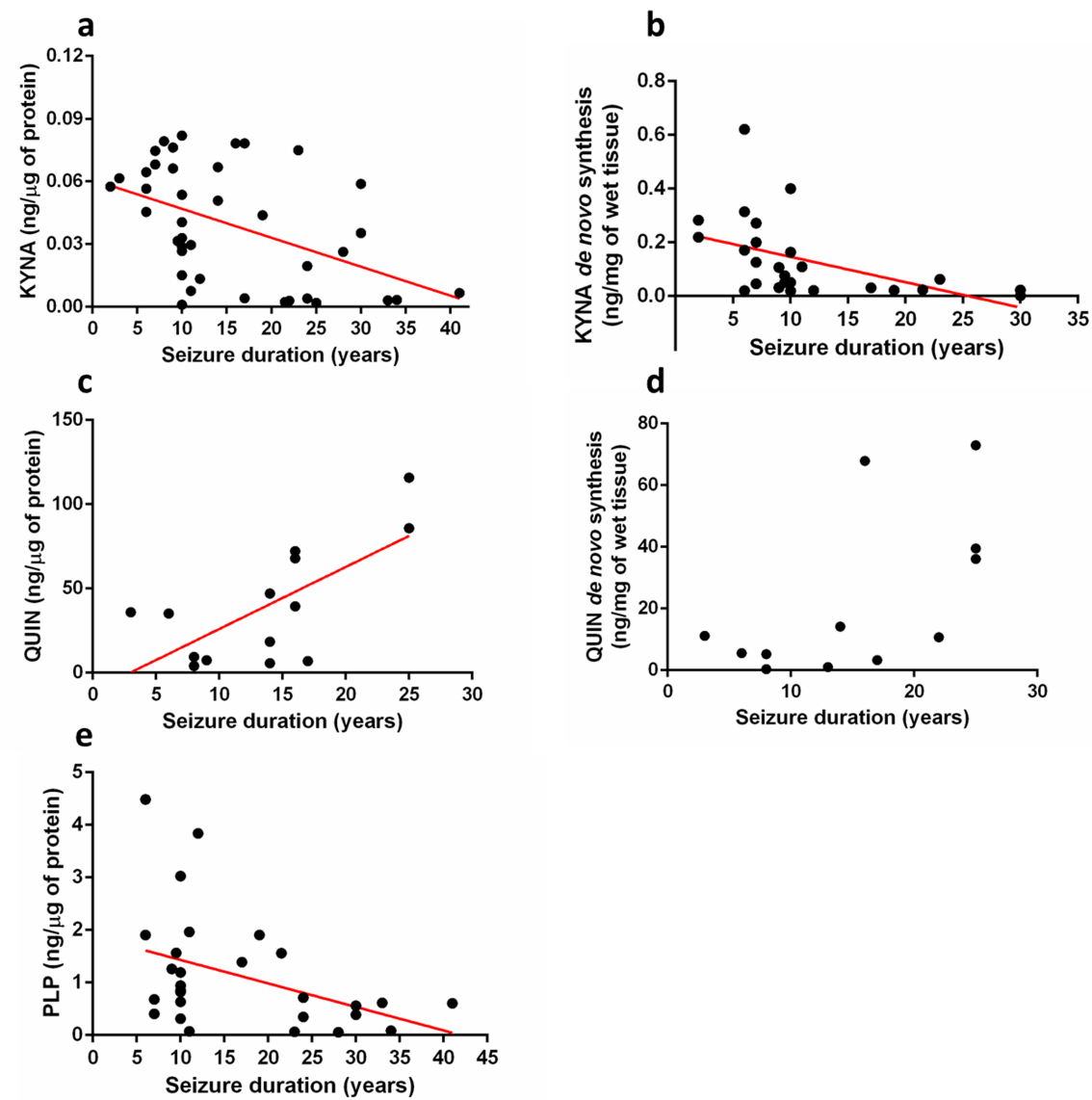

\section{Hosted file}

Table 1.pdf available at https://authorea.com/users/361994/articles/483248-alteredhippocampal-kynurenine-pathway-metabolism-contribute-to-hyperexcitability-in-mesialtemporal-lobe-epilepsy-hippocampal-sclerosis 